NASA Contractor Report 201631

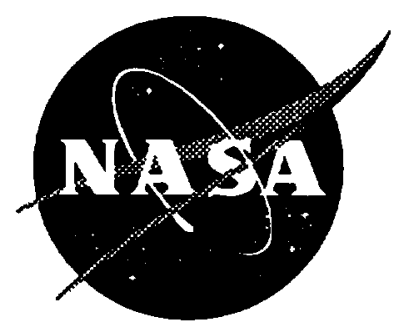

\title{
On the Coupling Between a Supersonic Turbulent Boundary Layer and a Flexible Structure
}

Abdelkader Frendi

Analytical Services $\mathcal{E}$ Materials, Inc., Hampton, Virginia

Contract NAS1-19700

October 1996

National Aeronautics and

Space Administration

Langley Research Center

Hampton, Virginia 23681-0001 


\title{
ON THE COUPLING BETWEEN A SUPERSONIC TURBULENT BOUNDARY LAYER AND A FLEXIBLE STRUCTURE
}

\author{
Abdelkader Frendi \\ Analytical Services \& Materials, Inc. \\ Hampton, VA 23666
}

\begin{abstract}
A mathematical model and a computer code have been developed to fully couple the vibration of an aircraft fuselage panel to the surrounding flow field, turbulent boundary layer and acoustic fluid. The turbulent boundary layer model is derived using a triple decomposition of the flow variables and applying a conditional averaging to the resulting equations. Linearized panel and acoustic equations are used. Results from this model are in good agreement with existing experimental and numerical data. It is shown that in the supersonic regime, full coupling of the flexible panel leads to lower response and radiation from the panel. This is believed to be due to an increase in acoustic damping on the panel in this regime. Increasing the Mach number increases the acoustic damping, which is in agreement with earlier work.
\end{abstract}

\section{Introduction}

Future civilian aircraft, subsonic or supersonic, will have to be quieter, more fuel efficient, less expensive and faster than today's aircraft. In light of these stringent requirements, a substantial amount of research and development work needs to be accomplished in various engineering fields. With the renewed interest in the development of a High Speed Civil Transport aircraft (HSCT), research interests have been increasing in the area of interior noise and sonic fatigue.

The source of aircraft interior noise is generally the vibrations of the outer skin of the fuselage. These vibrations are in turn caused by the pressure fluctuations in the mostly turbulent boundary layer on the aircraft. Therefore, in order to reduce the interior noise level, it is imperative that we understand the mechanisms by which noise is transmitted from the turbulent boundary layer to the interior. This is a challenging problem as it involves a fluid mechanics phenomenon, turbulence, that is the least understood in fluid mechanics.

Early work on the effects of turbulent boundary layer pressure fluctuations on structural vibration and interior noise generation focussed mainly on the structure and used either experimental data or empirical models to describe the pressure field in the turbulent boundary layer. One of the widely used models in the literature is the Corcos model [1]. 
This model was derived based on experimental observations and gives the cross spectral density of the pressure as;

$$
\Gamma(\zeta, \eta, \omega)=\Phi(\omega) A\left(\frac{\omega \zeta}{U_{c}}\right) B\left(\frac{\omega \eta}{U_{c}}\right) \exp \left(-i \frac{\omega \zeta}{U_{c}}\right)
$$

where $\Phi(\omega)$ describes the frequency content (or auto-spectrum), $A$ and $B$ the spatial distribution and the exponential term represents the convection of the pressure field. In the above equation, $\omega$ is the frequency, $\zeta$ and $\eta$ are the streamwise and spanwise separation distances and $U_{c}$ is the convection velocity. Based on the experiments of Willmarth and Wooldridge [2] the functions $A$ and $B$ were represented by decaying exponentials of the form;

$$
A\left(\frac{\omega \zeta}{U_{c}}\right)=\exp \left(-\alpha\left|\frac{\omega \zeta}{U_{c}}\right|\right) \quad \text { and } \quad B\left(\frac{\omega \eta}{U_{c}}\right)=\exp \left(-\beta\left|\frac{\omega \eta}{U_{c}}\right|\right)
$$

where the constants $\alpha$ and $\beta$ are arbitrary and are chosen to fit a given set of experimental data.

In recent years, many improvements of the original Corcos' model have been proposed [3-10]. Graham [11] performed a comparative study of the various models and concluded that for aircraft interior noise problems, Corcos' model is inadequate because of its inability to account for the dependence of the correlation length on boundary layer thickness. He recommended that the Efimtsov's extension of the Corcos model be used for such problems. The superior performance of the Efimtsov model was attributed to the fact that this model was derived from aircraft data rather than laboratory experiments. In addition to these models, several studies used the Monte Carlo method to solve this problem [1214]. This approach idealized the boundary layer pressure field to be a homogeneous, multidimentional Gaussian random process with zero mean.

It is important to emphasize that all of the above models need experimental data to determine the various constants and become useful. Since surface pressure data is difficult to obtain experimentally, especially for compressible high speed flows, the use of these models is further limited. In addition, once the constants are determined the pressure field in the turbulent boundary layer is fixed and the structural-fluid interaction problem is therefore decoupled. This approach can be useful in some engineering problems where coupling is not important (low speeds), however, at supersonic speeds the coupling between fluid and structure is very important. As was shown by Lyle and Dowell [15], acoustic damping on the structure becomes dominant as the speed is increased from subsonic to supersonic. This is further confirmed by the calculations of Wu and Maestrello [16] who showed that in a supersonic regime, neglecting the coupling term leads to an over-estimate of the structural response at higher modes.

In order to account for the various fluid-structure interaction effects, one needs to solve the complete set of partial differential equations for the fluid and the structure with the appropriate boundary conditions. The fluid motion is described by the nonlinear NavierStokes equations, and the structural response is given by the nonlinear plate equations. The computational requirements for solving such a problem will be dictated by the fluid equations. With the recent advancements in computers, the full Navier-Stokes equations 
have been integrated for a restricted number of flows and geometries. Such a method is referred to in the literature as Direct Numerical Simulation (DNS). This approach is severely limited by the range of Reynolds numbers that can be resolved and by the size of the computational domain. Therefore, its use in practical engineering problems is impossible at this point. The uses of this method have been restricted to generating data bases for some simple flows in order to validate various turbulence models being developed.

Since DNS is decades away from being a useful engineering tool, researchers in fluid dynamics have spent a great deal of time developing simplified forms of the Navier-Stokes equations. This effort has led to the development of a new approach known as the Large Eddy Simulation, LES. The difference between LES and DNS is in the number of resolved scales. In DNS, the grids used should be small enough to resolve all the scales down to the Kolmogorov scale; however, in LES the grids are coarser and the resolved scales are limited to those larger than the grid size. The contribution of the unresolved small scales (or subgrid scales) is modeled. One of the first LES models was introduced by Smagorinsky [17] in the early 1960s. This model performs reasonably well in free shear layers but does a poor job in wall bounded flows, i.e. boundary layers. Recently new models have been developed using the Smagorinsky idea but with more physical insight $[18,20]$. Though these models are more sophisticated, problems such as the kind of averaging one performs and how to treat the near-wall region, remain unresolved. In addition, these models need extensive testing before they can be applied to various engineering problems. From a computational view point, an LES calculation is one order of magnitude less expensive than an equivalent DNS calculation and the Reynolds number restriction is not as severe as it is for DNS. However, all these improvements over DNS do not make LES a preferred engineering tool for solving a large variety of problems. The weaknesses of LES are numerous; it is still too expensive computationally, it has yet to be used in complex geometries, spatial models are still in their infancy, etc.

Over a century ago, $O$. Reynolds [21] proposed a decomposition of the turbulent flow quantities into a time averaged mean, denoted by an overbar, and a fluctuation;

$$
f=\bar{f}+f^{\prime} .
$$

Using this decomposition, he derived a new set of equations for the mean quantities where the contribution from the turbulent fluctuations had to be modeled. These new equations became known as the Reynolds Averaged Navier-Stokes (RANS) equations and have been used to solve a wide variety of engineering problems. Since these equations are derived only for the mean quantities, they cannot provide any information on the dynamics of the flow. In order to overcome this deficiency, W.C. Reynolds and Hussain [22-24] used a triple decomposition of the form

$$
f=\bar{f}+\hat{f}+f^{\prime \prime}
$$

to study small amplitude wave disturbances in turbulent shear flows. In equation (4), $\hat{f}$ represents the wave motion and $f^{\prime \prime}$ is the turbulent fluctuation. A combination of time and conditional averaging were used to arrive at a set of dynamic equations describing the wave motion. Liu [25] used a similar approach to study the near-field jet noise due to the largescale wavelike eddies. Merkine and Liu [26] studied the development of noise-producing 
large-scale wavelike eddies in a plane turbulent jet. Liu and Merkine [27], Alper and Liu [28] and Gatski and Liu [29] studied the interactions between large-scale structures and fine-grained turbulence in a free shear flow. Gatski [30] calculated the sound production due to large-scale coherent structures in a free turbulent shear layer. More recently, Bastin et al. [31] used a semi-deterministic modeling approach coupled with Lighthill's acoustic analogy to calculate the jet mixing noise from unsteady coherent structures.

In the last few years, numerous advanced turbulence models have been developed which led to the solution of a wide variety of engineering problems. The extension of the RANS method to unsteady problems has been made less difficult with the new models because they contain more physics. The unsteady RANS method has been identified by many researchers as being equivalent to a Very Large Eddy Simulation or VLES [32]. The advantages of using VLES are: it is computationally less costly than either LES or DNS, the models have been tested extensively and the method can be used for any geometry, etc.

The remainder of this paper is organized as follows; in section (2) the mathematical model is described, section (3) describes the method of solution used to solve the model, the results and discussions are given in section (4) and a summary of the results and some concluding remarks are given in section (5).

\section{Mathematical Model}

\subsection{The Turbulent Boundary Layer Equations}

Using the triple decomposition proposed by W.C. Reynolds [24], the flow quantities are decomposed as follows;

$$
g=\tilde{g}+\hat{g}+g^{\prime \prime}
$$

where $g$ represents a flow quantity and $(\tilde{g})$ its Favre averaged mean defined by

$$
\bar{g}=\frac{\overline{(\rho g)}}{\bar{\rho}} .
$$

When decomposing the density, the turbulent fluctuations, $\rho$ ", are neglected by virtue of Morkovin's hypothesis [33] which has been recently verified by Sommer et al. [34], therefore;

$$
\rho=\bar{\rho}+\hat{\rho} .
$$

In equations $(4)$ and $(6),(\hat{g}, \hat{\rho})$ is the low frequency variation part of the mean and $\left(g^{\prime \prime}\right)$ is the turbulent fluctuation.

By defining the total mean to be

$$
G=\tilde{g}+\hat{g},
$$

equation (4) becomes

$$
g=G+g^{\prime \prime}
$$


Equation (8) has a similar form to equation (3); the only difference is that in (3), $\bar{f}$ is a time independent mean while in (8), $G$ is time dependent.

In the derivation of the dynamic equations used by W.C. Reynolds, a conditional averaging was introduced. Some properties of this averaging are;

$$
\begin{gathered}
<g^{\prime \prime}>=0 \quad\langle\hat{g} f>=\hat{g}<f> \\
<\tilde{g} f>=\tilde{g}<f>\quad \widetilde{\langle g}>=\tilde{g}=<\tilde{g}> \\
\widetilde{\hat{g} f^{\prime \prime}}=<\widetilde{\hat{g} f^{\prime \prime}}>=0 .
\end{gathered}
$$

Using the decomposition given by equation (8) in the continuity, momentum, energy and state equations along with the conditional averaging and Einstein summation convention one arrives at the following mass, momentum, energy and state equations;

$$
\begin{gathered}
\frac{\partial \rho}{\partial t}+\frac{\partial}{\partial x_{i}}\left(\rho U_{i}\right)=0, \quad i=1,2,3 \\
\frac{\partial}{\partial t}\left(\rho U_{i}\right)+\frac{\partial}{\partial x_{j}}\left[\rho U_{i} U_{j}-<\tau_{i j}>+\rho<u_{i}^{\prime \prime} u_{j}^{\prime \prime}>\right]+\frac{\partial P}{\partial x_{i}}=0, \quad i, j=1,2,3 \\
\frac{\partial E}{\partial t}+\frac{\partial}{\partial x_{j}}\left[(E+P) U_{j}-<\tau_{i j}>U_{j}+<e^{\prime \prime} u_{j}^{\prime \prime}>+<p^{\prime \prime} u_{j}^{\prime \prime}>-<\tau_{i j} u_{j}^{\prime \prime}>\right]=0 \\
P=(\gamma-1)\left[E-\rho\left(U_{1}^{2}+U_{2}^{2}+U_{3}^{2}+<\left(u_{1}^{\prime \prime}\right)^{2}>+<\left(u_{2}^{\prime \prime}\right)^{2}>+<\left(u_{3}^{\prime \prime}\right)^{2}>\right) / 2\right] .
\end{gathered}
$$

In equations (10)-(13), $\left(\rho, U_{i}, E, P\right)$ represent the total means of the variables $\left(\rho, u_{i}, e, p\right)$, while $\left(u_{i}^{\prime \prime}, u_{j}^{\prime \prime}, e^{\prime \prime}, p^{\prime \prime}\right)$ are the turbulent fluctuations. The variable $(e)$ is the total energy defined as $e=p /(\gamma-1)+\rho\left(u_{1}^{2}+u_{2}^{2}+u_{3}^{2}\right) / 2$. In equations (11) and (12), $\left\langle\tau_{i j}\right\rangle$ is the conditionally averaged stress tensor,

$$
<\tau_{i j}>=\frac{M_{\infty}}{R e_{L}}\left[\mu\left(\frac{\partial U_{i}}{\partial x_{j}}+\frac{\partial U_{j}}{\partial x_{i}}\right)-\frac{2}{3} \mu \frac{\partial U_{k}}{\partial x_{k}} \delta_{i j}\right],
$$

where $M_{\infty}, R e_{L}$ and $\mu$ are the freestream Mach number, Reynolds number and molecular viscosity, respectively. The term $\left\langle u_{i}^{\prime \prime} u_{j}^{\prime \prime}\right\rangle$ of equation (11) is similar to the Reynolds stress tensor and is modeled in the same way. In equation (12), $\left\langle e^{\prime \prime} u_{j}^{\prime \prime}\right\rangle,\left\langle p^{\prime \prime} u_{j}^{\prime \prime}\right\rangle$ and $\left\langle\tau_{i j} u_{j}^{\prime \prime}\right\rangle$ have to be modeled.

Invoking the Boussinesq approximation that the Reynolds stress tensor is proportional to the mean strain-rate tensor, leads to

$$
\rho<u_{i}^{\prime \prime} u_{j}^{\prime \prime}>=\frac{2}{3} \rho k \delta_{i j}-2 \mu_{t}\left(S_{i j}-\frac{1}{3} S_{k k} \delta_{i j}\right)
$$

where $k=\left\langle u_{i}^{\prime \prime} u_{i}^{\prime \prime}\right\rangle / 2$ is the turbulent kinetic energy, $\mu_{t}$ is the turbulent eddy viscosity and $S_{i j}$ is the mean strain-rate tensor given by,

$$
S_{i j}=\frac{1}{2}\left[\frac{\partial U_{i}}{\partial x_{j}}+\frac{\partial U_{j}}{\partial x_{i}}\right] .
$$


The turbulent eddy viscosity is obtained from the relation

$$
\mu_{t}=C_{\mu}^{\star} \frac{\rho k}{\omega}
$$

where $C_{\mu}^{\star}$ is a constant, $\omega$ the specific dissipation rate $(\epsilon / k)$ with $\epsilon$ being the dissipation. The conservation equations for $k$ and $\omega$ are;

$$
\begin{gathered}
\frac{\partial}{\partial t}(\rho k)+\frac{\partial}{\partial x_{j}}\left(\rho U_{j} k\right)=-\rho<u_{i}^{\prime \prime} u_{j}^{\prime \prime}>\frac{\partial U_{i}}{\partial x_{j}}-\rho \omega k+\frac{\partial}{\partial x_{j}}\left(\frac{\mu_{t l}}{\sigma_{k}} \frac{\partial k}{\partial x_{j}}\right) \\
\frac{\partial}{\partial t}(\rho \omega)+\frac{\partial}{\partial x_{j}}\left(\rho U_{j} \omega\right)=-\rho C_{\omega_{1}} \frac{\omega}{k}\left\langle u_{i}^{\prime \prime} u_{j}^{\prime \prime}>\frac{\partial U_{i}}{\partial x_{j}}-C_{\omega_{2}} \rho \omega^{2}+\frac{\partial}{\partial x_{j}}\left(\frac{\mu_{t l}}{\sigma_{\omega}} \frac{\partial \omega}{\partial x_{j}}\right) .\right.
\end{gathered}
$$

In equations (18) and (19), $\mu_{t l}=C_{\mu l}^{\star} \rho(k / \omega)$ and $C_{\omega_{1}}=C_{\omega_{2}}-\kappa^{2} /\left(\left(C_{\mu l}^{\star}\right)^{0.5} \sigma_{\omega}\right)$, with $C_{\mu l}^{\star}=0.09$ (same as $C_{\mu}^{\star}$ in the $\log$ layer), $\sigma_{k}=\sigma_{\omega}=2, C_{\omega_{2}}=0.83$ and $\kappa=0.41$. The model described above was derived by Wilcox [35] and is known as the $(k-\omega)$ turbulence model.

\subsection{The Plate Equation}

The out-of-plane plate displacement, $w$, is given by the biharmonic equation,

$$
D \Delta^{2} w+\rho_{p} h \frac{\partial^{2} w}{\partial t^{2}}+\Gamma \frac{\partial w}{\partial t}=\delta p
$$

where $D$ is the plate stiffness obtained from $D=E_{p} h^{3} / 12\left(1-\nu_{p}^{2}\right)$, with $E_{p}$ being the Young Modulus, $h$ the plate thickness and $\nu_{p}$ the Poisson ratio. In equation (20), $\rho_{p}$ is the plate material density and $\Gamma$ is the structural damping. The biharmonic term is defined as

$$
\Delta^{2}=\frac{\partial^{4}}{\partial x^{4}}+2 \frac{\partial^{4}}{\partial x^{2} \partial y^{2}}+\frac{\partial^{4}}{\partial y^{4}}
$$

The right hand side, $\delta p$, of equation (20) represents the pressure loading due to the adjacent fluids and can be written as

$$
\delta p=p^{a}-p^{b l}
$$

with $\left(p^{a}\right)$ being the radiated acoustic pressure and $\left(p^{b l}\right)$ the turbulent boundary layer pressure calculated from the model in section (2.1).

\subsection{The Acoustic Radiation Equation}

In order to calculate the pressure $p^{a}$ of equation (22), Kirchhoff's formula is used to arrive at (see [36] for details)

$$
p^{a}(x, y, z, t)=\frac{\rho_{\infty}}{2 \pi} \iint_{D} \frac{\left[w_{t t}\left(\tau, x^{\prime}, z^{\prime}\right)\right]}{R} d x^{\prime} d z^{\prime}
$$


where the integration domain, $D$, is the whole plate and $w_{t t}=\frac{\partial^{2} w}{\partial t^{2}}$. In equation (23), the square brackets, [.], are used to denote a retarded time, i.e.

$$
\left[w_{t t}\left(\tau, x^{\prime}, z^{\prime}\right)\right]=w_{t t}\left(t-R / c_{\infty}, x^{\prime}, z^{\prime}\right)
$$

where $R=\left[\left(x-x^{\prime}\right)^{2}+y^{2}+\left(z-z^{\prime}\right)^{2}\right]^{1 / 2}$ is the distance from an observer point $(x, y, z)$ to a point on the plate $\left(x^{\prime}, 0, z^{\prime}\right)$. In equation (24), $c_{\infty}$ is the speed of sound. Equation (23) is used to calculate the pressure both on the surface of the plate and in the far-field. When using equation (23) to calculate the surface pressure, a Taylor series expansion of the integrand is used to avoid the singularity at $R=0$ (when the observer point coincides with the source).

\section{Method of Solution}

The turbulent boundary layer equations are solved using the three-dimensional thinlayer Navier-Stokes code known as CFL3D [37]. The numerical method uses a second order accurate finite volume scheme. The convective terms are discretized with an upwind scheme that is based on Roe's flux difference splitting method, while all the viscous terms are centrally differenced. The equations are integrated in time with an implicit, spatially split approximate-factorization scheme. The thin-layer approximation retains only those viscous terms with derivatives normal to the body surface. This is generally considered to be a good approximation for high-Reynolds-number aerodynamic flows with minimal separation. Two calculations are made for each case; first the steady state mean velocity profiles are obtained using a large domain that includes the leading edge of the plate, then using a smaller domain downstrean of the leading edge an unsteady calculation is carried out by perturbing the mean velocity profile at the inflow boundary as follows

$$
u=\tilde{u}+\epsilon R_{n}(y, z, t)
$$

In equation (25), $R_{n}(y, z, t)$ is a random number generated using an IMSL routine called RNNOF [38] and $\epsilon$ is a small amplitude chosen to be between 0.05 and 0.25 . In the steady state calculation, the flow in the region upstream of the plate's leading edge is specified as laminar, while that downstream of the leading edge is turbulent. The plate equation (20) is integrated using an implicit finite difference method for structural dynamics developed by Hoff and Pahl [39]. The radiated acoustic pressure, $p^{a}$, is obtained through a combination of Simpson's integration rule in space and a trapezoidal rule in time.

Coupling between the plate and the acoustic and boundary layer pressure fields is obtained as follows. Using the previous time step plate velocity and acceleration as boundary conditions, the turbulent boundary layer equations (10)-(13), (18), (19) and the acoustic equation (23) are integrated to obtain the new surface pressure fields, these are then used

to update the plate equation. This procedure is repeated at every time step. Figure 1 shows a two dimensional computational domain with the different mathematical models. The top domain shows the presence of a leading edge shock, because all the cases studied are supersonic. In the following results, the flexible plate is clamped between two rigid ones except in one case where the plate is simply supported. 


\section{Results and Discussions}

This section is divided into several subsections. In the subsection (4.1) results from a typical fully coupled run are presented. Comparisons to existing experimental data are given in subsection (4.2). In subsection (4.3), the effects of coupling on the plate response and radiation are discussed. In subsection (4.4), results from two runs at two different Mach numbers are given and finally in subsection (4.5) the effects of plate boundary conditions on the response and acoustic radiation are analyzed.

\subsection{Results From a Typical Fully Coupled Case}

The flow and structural properties used in this case are taken from Maestrello [40]. The mean flow parameters are; $M_{\infty}=1.98$ (free-stream Mach number), $T_{t}=563 \mathrm{R}$ (Total temperature), $T_{w}=550 \mathrm{R}$ (Wall temperature) and $R e / f t=3.73 \times 10^{6}$ (Reynolds number per foot). A titanium plate is used in this study with the following parameters; length, $a=12$ inches, width $b=6$ inches, thickness $h=0.062$ inches, density per unit area $\rho_{p} h=2.315 \times 10^{-5} \mathrm{lbf} \cdot \sec ^{2} / \mathrm{in}^{3}$, stiffness $D=345$. lbf.in and damping $\Gamma=7.4 \times 10^{-4}$ $\mathrm{lbf} \cdot \mathrm{sec} / \mathrm{in}^{3}$. The parameter $\epsilon$ of equation (25) is set to 0.25 . The plate is oriented such that the length, $a$, is along the streamwise direction.

The computational domain used is $2 \times 1 \times 2 f t$ in the streamwise, spanwise and vertical directions, respectively. the number of points used in the respective directions are $83 \times 53 \times 83$. Equally spaced points are used in the streamwise and spanwise directions while grid stretching is used in the vertical direction. The leading edge of the plate is 0.5 $f t$ upstream of the inflow boundary. For each case studied, the second grid point in the vertical direction is located at a $y^{+}<1$., where $y^{+}$is a nondimensional coordinate given by, $y^{+}=y u_{\tau} / \nu$ with $u_{\tau}=\left(\tau_{w} / \rho\right)^{0.5}$ being the friction velocity, $\nu$ the kinematic viscosity and $\rho$ the density. In the $u_{\tau}$ expression, $\tau_{w}$ is the wall shear stress.

Figures 2a-2c show the time histories of the turbulent boundary layer surface pressure at the center of the flexible plate, the center plate displacement and the radiated pressure one foot away from the plate center, respectively. The time history of the turbulent boundary layer pressure, Fig. 2a, shows a random fluctuation around the mean pressure $\bar{p}_{r e f}$. The displacement time history shows the presence of several frequencies but with one dominant low frequency, Fig. 2b. The time history of the radiated pressure is also random around the mean pressure, Fig. 2c. The corresponding power spectral densities (PSD) are shown on Figs. 3a-3c. The PSD of the turbulent boundary layer pressure shows the presence of a broad peak at around $5000 \mathrm{~Hz}$, Fig. 3a. The level decreases slowly with decreasing frequency from the peak, while above $5000 \mathrm{~Hz}$ the level decreases sharply with increasing frequency. Grid refinement and time step refinement show little effect on the location of the peak. The PSD of the displacement response at the center of the plate, Fig. 3b, shows the presence of several peaks corresponding to the various response modes of the plate. This response, as the figure shows, is dominated by the first mode which is the $(1,1)$ mode located near $430 \mathrm{~Hz}$. Figure 3c shows the PSD of the radiated pressure one foot away from the center of the plate. The frequency content of this radiated field is dominated by two plate mode frequencies, one corresponding to the $(1,1)$ mode and 
the other to the $(5,1)$ mode. Other intermediate frequencies are also present but at a much lower level. This result is of great significance from a noise control point of view. This means that controlling the vibration of the plate at a few natural frequencies, can reduce the noise level significantly. The probability distributions of the input pressure, Fig. 4a, the displacement response, Fig. 4b, and the radiated pressure, Fig. 4c, show a Gaussian like behavior which is consistent with the time histories shown on Figs. 2a-2c. The cross-correlation function of the surface pressure is defined as

$$
R_{p p}(\zeta, \eta, \tau)=\frac{\overline{p(x, z, t) p(x+\zeta, z+\eta, t+\tau)}}{\bar{p}^{2}}
$$

where $\zeta$ and $\eta$ are the steamwise and spanwise separation distances, and $\tau$ the time separation. The streamwise two-point correlation is obtained from equation (26) by setting $(\eta, \tau)$ to zero. Similarly, the spanwise two-point correlation and the auto-correlation are obtained by setting $(\zeta, \tau)$ and $(\zeta, \eta)$ to zero, respectively. Figures $5 \mathrm{a}-\mathrm{b}$ show the streamwise and spanwise two-point correlations. Both figures show that as the separation distance increases, the correlation function decreases rapidly to nearly zero indicating the lack of correlation between the pressures at various points. Figure 6 shows the auto-correlation as a function of time. The auto-correlation decreases to zero very rapidly as time increases. Both the two-point correlations and auto-correlation behave in a manner that is characteristic of a turbulent boundary layer. Figure 7 shows a complicated instantaneous displacement response of the plate.

\subsection{Comparisons to Experiments}

Maestrello's data [40] is used for the various comparisons. The mean flow parameters used are; Mach number $M_{\infty}=3.03$, total temperature $T_{t}=567 \mathrm{R}$ and a Reynolds number per foot $R e / f t=4.265 \times 10^{6}$. The plate parameters are the same as those given in subsection (4.1), and the parameter $\epsilon$ is set to 0.25 . Figure 8 shows the mean velocity profile $\left(u^{+}=u / u_{\tau}\right.$ versus $\left.y^{+}=y u_{\tau} / \nu\right)$. As shown on the figure, there is no experimental data point for $y^{+}$less than 60 , this is due to the difficulty in making near wall measurements. The agreement between Maestrello's data and the numerical results in the logarithmic layer region is good. In addition the profile shows a linear behavior in the sub-layer region near the wall which is a characteristic of a turbulent boundary layer velocity profile. Figure 9 shows the PSD of the center plate displacement response obtained experimentally and numerically. The experimental results show that the response is dominated by the $(1,1)$ and $(3,1)$ modes. The numerical results also show the dominance of the same two modes. The agreement between the numerical and experimental results is good at the lower modes; however the numerical results overpredict the response at higher modes. This is due to the numerical technique used to integrate the plate equation and the number of grid points used on the plate. The higher modes are less resolved and therefore show a higher response.

Figure 10 shows the PSDs of the surface pressure measured at the center of the flexible plate and that calculated numerically. The PSD of the pressure measured experimentally shows and increase in level as the frequency increases until a broad peak is reached in the vicinity of $5000 \mathrm{~Hz}$. At high frequencies (> $10000 \mathrm{~Hz}$ ) the level decreases slowly. The numerical results show a similar behavior at low frequencies; however, at high frequencies 
( $>6000 \mathrm{~Hz}$ ) the level decreases rapidly. This rapid decrease in level is believed to be due to both numerical resolution and turbulence model used. Higher grid resolution and an advanced turbulence model would result in better agreement at high frequencies. Recent experimental measurements on the Concorde [41] show similar behavior of the surface pressure fluctuation to that of Maestrello [40]. The frequency of the peak level depended on the measurement's location on the fuselage. It is important to mention at this point that based on the results presented in subsection 4.1, the frequencies that are critical for the structure are in the range up to $2000 \mathrm{~Hz}$. The numerical results of Fig. 9 show a good agreement with experiments for this frequency range. The parameter $\epsilon$ of equation (25) can be adjusted to obtain better agreement with experimentally determined levels.

\subsection{Effect of Coupling}

The mean flow and plate properties used in this calculation are the same as those given in subsection (4.1). In order to access the effects of full coupling on the response of a flexible plate, two calculations are carried out. In one calculation, the turbulent boundary layer, the radiated acoustic field and the flexible plate are fully coupled. The plate communicates on both sides with the surrounding fluid. In the other calculation, the turbulent boundary layer pressure field is directly transmitted to the plate at each time step; however the plate response is not communicated back into the fluid flow. This latter case is referred to as uncoupled. Figure 11 shows the PSD of the turbulent boundary layer pressure fluctuations at the plate center for both the coupled and uncoupled cases. There is little or no difference between the two PSDs. This is expected since the plate vibration has little effect on the turbulent boundary layer. The PSD of the displacement response at the center of the plate is shown on Fig. 12 for the two cases. It is important to notice that the response of the uncoupled plate is higher through the whole frequency range. This is an indication that coupling results in more damping on the structure and therefore less response. Based on the results obtained by Frendi and Maestrello [42], this difference would be even greater at higher excitation levels where shifting in the peak responses occurs. As expected the higher plate response in the uncoupled case results in higher noise levels, as shown in Fig. 13. This result is important for interior noise considerations. In other words, an uncoupled plate analysis significantly overpredicts interior noise levels, particularly at the lower frequencies where noise control is typically both difficult and expensive in terms of added weight. Therefore one needs to consider the response of a fully coupled plate before deciding on noise reduction techniques to be used.

\subsection{Effect of Mach Number}

Two Mach number cases have been studied, 1.98 and 3.03, using the mean flow properties given in the above subsections. Figure 14 shows the PSD of the surface pressure at the center of the plate for the two cases. The two PSDs are nearly identical. The PSDs of the displacement response at the plate center show little or no difference between the two cases at low frequencies. However at high frequencies, the response for $M_{\infty}=1.98$ is higher, Fig. 15. This is attributed to an increase in acoustic damping with increasing Mach number. This result is consistent with the results of Frendi and Maestrello [42] and Lyle and Dowell [43] who showed that increasing the Mach number increases the acoustic 
damping on the structure. Figure 16 shows the PSDs of the radiated pressure for the two cases. Since there is little or no difference in the response at low frequencies, the radiated pressure PSDs are nearly identical.

\subsection{Effect of Plate Edge Conditions}

In order to access the effect of edge conditions on plate vibration and acoustic radiation, two calculations are made using the same mean flow properties as in subsection (4.1). Figure 17 shows that the PSDs of the pressure at the center of the plate from the turbulent boundary layer side are nearly identical. However, the PSD of the plate displacement response shows a different frequency content as expected, Fig. 18. The simply supported plate shows lower natural frequencies and a higher response at the lowest modal frequency. The PSDs of the radiated pressure also show a shift in the frequency content to lower frequencies for the simply supported case. A slightly lower level is also indicated and is due mainly to lack of frequency resolution, Fig. 19. The objective of this calculation is to show the direct effect of changing any plate parameter on the radiated pressure field. Additional calculations involving changing other plate properties (such as mass, stiffness, damping...etc) can be made using this approach in order to analyze their effect on the radiation field.

\section{Concluding Remarks}

A model that couples a supersonic turbulent boundary layer with a flexible plate and the radiated acoustic field has been developed. A three dimensional code has been written to solve this model. The code is a modified version of CFL3D. Extensive two dimensional and three dimensional test calculations have been carried out. Figure 20 summarizes the capability of the code. The results given by this model show good agreement with existing experimental results both for the structural response and pressure fluctuations in the turbulent boundary layer. Additional results showed that;

- Full coupling of the plate is important for accurate response and acoustic radiation calculations.

- As the Mach number increases the acoustic damping on the plate increases. The acoustic damping lowers the response especially high modes.

- Changing the boundary conditions of the plate changes the response and the radiated pressure frequency content. This shows that a computational tool can be used to assess the best noise reduction mechanisms. Further test runs can be made to support this idea.

\section{Acknowledgements}

The author would like to acknowledge the support of the Structural Acoustics Branch of NASA Langley Research Center under contract NAS1-19700. I would like to thank Dr. Thomas Gatski for his availability to discuss the problem and the helpful advice he gave me during the course of this work. 


\section{References}

[1] Corcos, G.M., "Resolution of pressure in turbulence," The Journal of the Acoustical Society of America, vol. 35, no. 2, pp. 192-198, 1963.

[2] Willmarth, W.W., and Wooldridge, C.E., "Measurements of the fluctuating pressure at the wall beneath a thick turbulent boundary layer," Journal of Fluid Mechanics, vol. 14, pf. 187, 1962.

[3] Ffowcs Williams, J.E., "Boundary layer pressures and the Corcos model: a development to incorporate low wavenumber constraints", Journal of Fluid Mechanics, vol. 125, pp. 9-25, 1982.

[4] Chase, D.M., "Modeling the wavenumber-frequency spectrum of turbulent boundary layer wall pressure," Journal of Sound and Vibration, vol. 70, no. 1, pp. 29-67, 1980.

[5] Chase, D.M., "The character of the turbulent wall pressure spectrum at the subconvective wavenumbers and a suggested comprehensive model," Journal of Sound and Vibration, vol. 112, no. 1, pp. 125-147, 1987.

[6] Laganelli, A.L., Martellucci, A., and Shaw, L.1., "Wall pressure fluctuations in attached boundary layer flows," AIAA Journal, vol. 21, no. 4, 1983.

[7] Laganelli, A.L., and Wolfe, H., "Prediction of fluctuating pressure in attached and separated turbulent boundary layer flow," AIAA paper 89-1064. The 12th AIAA Aeroacoustics conference, San Antonio, TX, 1989.

[8] Blake, W.K., "Turbulent boundary layer wall pressure fluctuations on smooth and rough walls," Journal of Fluid Mechanics, vol. 44, part 4, 1970.

[9] Smol'yakov, A.V., and Tkachenko, V.M., "Model of a field of pseudosonic turbulent wall pressures and experimental data," Soviet Physical Acoustics, vol. 37, no. 6 , pp. 627-631, 1991.

[10] Efimtsov, B.M., "Characteristics of the field of turbulent wall pressure fluctuations at large Reynolds numbers," Soviet Physical Acoustics, vol. 28, no. 4, pp. 289-292, 1982.

[11] Graham, W.R., "A comparison of models for the wavenumber frequency spectrum of turbulent boundary layer pressures," Proceedings of the first AIAA/CEAS conference on aeroacoustics, Munich, Germany, pp. 711-720, 1994.

[12] Dowell, E.H., "Generalized aerodynamic forces on a flexible plate undergoing transient motion," Q. Applied Mathematics, vol. 24, pp. 331-338, 1967.

[13] Shinozuka, M., "Simulation of multivariate and multidimensional random processes," The Journal of the Acoustical Society of America, vol. 47, no. 1, part 2, pp. 357-367, 1971.

[14] Vaicaitis, R., Jan, C.M., and Shinozuka, M., "Nonlinear panel response from a 
turbulent boundary layer", AIA A Journal, vol. 10, no. 7, pp. 895-899, 1972.

[15] Lyle, K.H. and Dowell, E.H., "Acoustic radiation damping of rectangular composite plates subjected to subsonic flows," Journal of Fluids and Structures, to appear.

[16] Wu, S.F. and Maestrello, L., "Responses of finite baftled plate to turbulent flow excitations," AIAA Journal, vol. 33, no. 1, pp. 13-19, 1995.

[17] Smagorinski, J., "General circulation experiments with the primitive equations. I. The basic experiments," Mon. Weather Rev., vol. 91, pf. 99, 1963.

[18] Moin, P., and Kim, J., "Numerical investigation of turbulent channel flow," Journal of Fluid Mechanics, vol. 118, pf. 381, 1982.

[19] Germano, M., Piomelli, U., Moin, P., and Cabot, W.H., "A dynamic subgrid scale eddy viscosity model," Physics of Fluids A, vol. 3, pf. 1760, 1991.

[20] El-hady, N.M., Zang, T.A., and Piomelli, U., "Application of the dynamic subgridscale model to axisymmetric transitional boundary layer at high speed," Physics of Fluids, vol. 6, no. 3, pp. 1299-1309, 1994.

[21] Reynolds, O., Philosophical Transactions of the Royal Society of London, vol. 174, pf. 935, 1883.

[22] Hussain, A.K.M.F., and Reynolds, W.C., "The mechanics of an organized wave in turbulent shear flow", Journal of Fluid Mechanics, vol. 41, part 2, pp. 241-258, 1970.

[23] Hussain, A.K.M.F., and Reynolds, W.C., "The mechanics of an organized wave in turbulent shear flow. Part 2: Experimental Results," Journal of Fluid Mechanics, vol. 54, part 2, pp. 241-261, 1972.

[24] Reynolds, W.C., and Hussain, A.K.M.F., "The mechanics of an organized wave in turbulent shear flow. Part 3: Theoretical models and comparisons with experiments," Journal of Fluid Mechanics, vol. 54, part 2, pp. 263-288, 1972.

[25] Liu, J.T.C., "Developing large-scale wavelike eddies and the near jet noise field," Journal of Fluid Mechanics, vol. 62, part 3, pp. 437-464, 1974.

[26] Merkine, L., and Liu, J.T.C., "On the development of noise-producing large-scale wave-like eddies in a plane turbulent jet," Journal of Fluid Mechanics, vol. 70, part 2, pp. 353-368, 1975.

[27] Liu, J.T.C., and, Merkine, L., "On the interactions between large-scale structure and fine-grained turbulence in a free shear flow. I. The development of temporal interactions in the mean," Proceedings of the Royal Society of London, vol. 352, pp. 213-247, 1976.

[28] Alper, A., and Liu, J.T.C., "On the interactions between large-scale structure and fine-grained turbulence in a free shear flow. II. The development of spatial interactions in the mean," Proceedings of the Royal Society of London A., vol. 359, pp. 497-523, 1978. 
[29] Gatski, T.B., and Liu, J.T.C., "On the interactions between large-scale structure and fine-grained turbulence in a free shear flow. III. A numerical solution," Proceedings of the Royal Society of London A., vol. 293, pf. 473, 1980.

[30] Gatski, T.B., "Sound production due to large-scale coherent structures," AIAA Journal, vol. 17, no. 6, pp. 614-621, 1979.

[31] Bastin, F., Lafon, P., and Candel, S., "Computation of jet mixing noise from unsteady coherent structures," CEAS/AIAA paper 95-039, 1995.

[32] Orzag, S.A., "Turbulent flow modeling and prediction," Institute for Computer Applications in Sciences and Engineering (ICASE) and Langley Research Center short course, March 14-18, 1994.

[33] Morkovin, M., "Effects of compressibility on turbulent flows," Mecanique de la turbulence, CNRS, edited by A. Favre, published by Gordon and Breach, pp. 367-380, 1962.

[34] Sommer, T.P., So, R.M.C., and Gatski, T.B., "Verification of Morkovin's hypothesis for the compressible turbulence field using direct numerical simulation data," AIAA paper 95-0859, 33rd Aerospace Sciences Meeting and Exhibit, Reno, NV, 1995.

[35] Wilcox, D.C., "Reassessment of the scale-determining equation for advanced turbulence models," AIAA Journal, vol. 26, no. 11, pp. 1299-1310, 1988.

[36] Frendi, A., Maestrello, L., and Ting, L., "An efficient model for coupling structural vibrations with acoustic radiation," Journal of Sound and Vibration, vol. 182 , no. 5, pp. 741-757, 1995.

[37] Rumsey, C., Thomas, J., Warren, G., and Liu ,G., "Upwind Navier-Stokes solutions for separated periodic flows," AIAA paper 86-0247, 1986.

[38] International Mathematical and Statistical Library (IMSL), version 2.0, vol. 3, Houston, TX, pf. 1317, 1991.

[39] Hoff, C., and Pahl, P.J., "Development of an implicit method with numerical dissipation from a generalized single-step algorithm for structural dynamics," Comp. Meth. Appl. Mech. Eng., vol. 67, no. 2, pp. 367-385, 1988.

[40] Maestrello, L., "Radiation from and panel response to a supersonic turbulent boundary layer," Journal of Sound and Vibrations, vol. 10, no. 2, pp. 261-295, 1969.

[41] Goodwin, P.W., "An in-flight supersonic turbulent boundary layer surface pressure fluctuation model," Boeing document no. D6-81571, 1994.

[42] Frendi, A., and Maestrello, L., "On the combined effect of mean and acoustic excitation on structural response and radiation," Journal of Vibration and Acoustics, in press.

[43] Lyle, K.H., and Dowell, E.H., "Acoustic radiation damping of flat rectangular plates subjected to subsonic flows," Journal of Fluids and Structures, in press. 


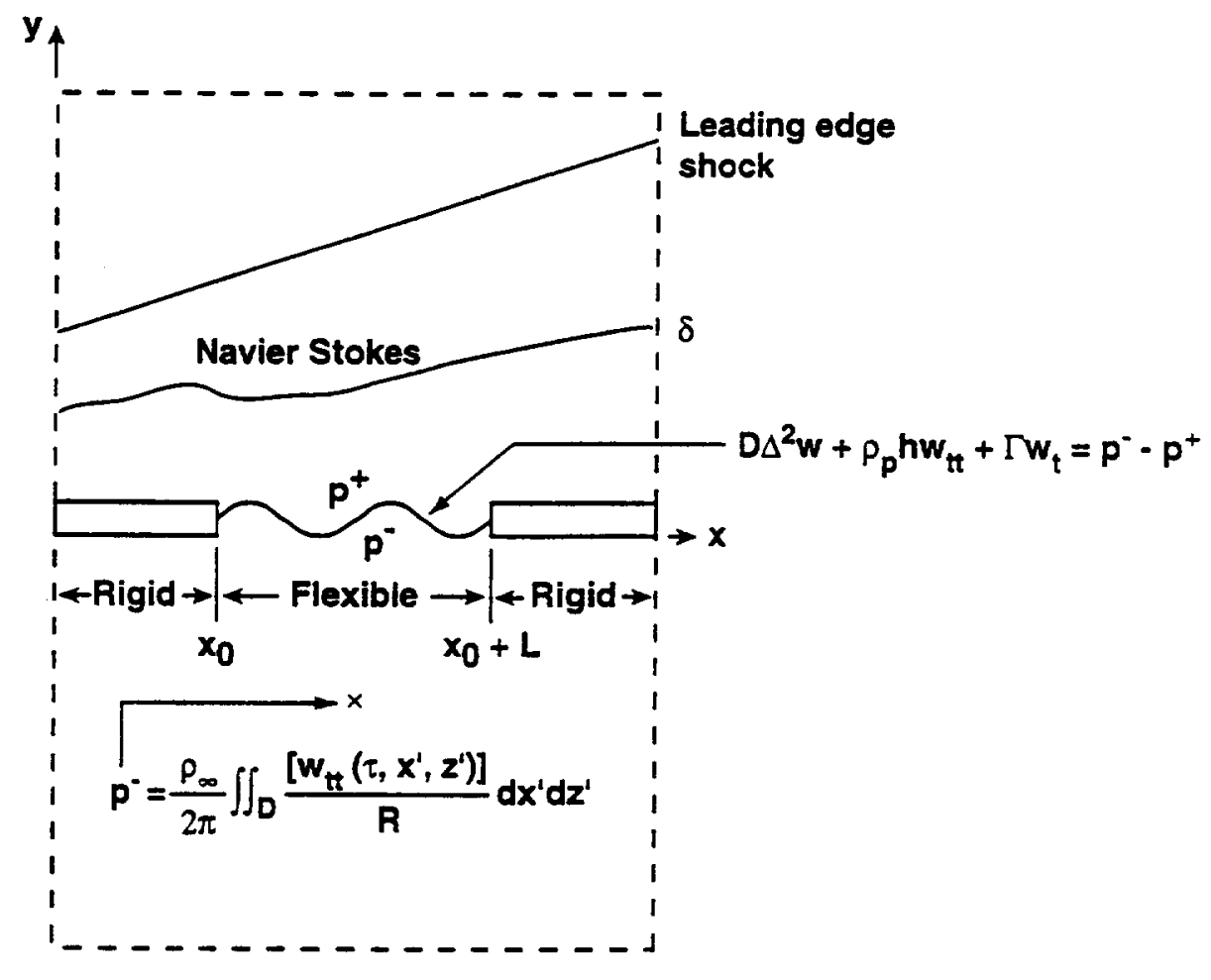

Figure 1: Two dimensional computational domain and the mathematical model. 




Figure 2a: Time history of the turbulent boundary layer surface pressure at the center of the plate. 


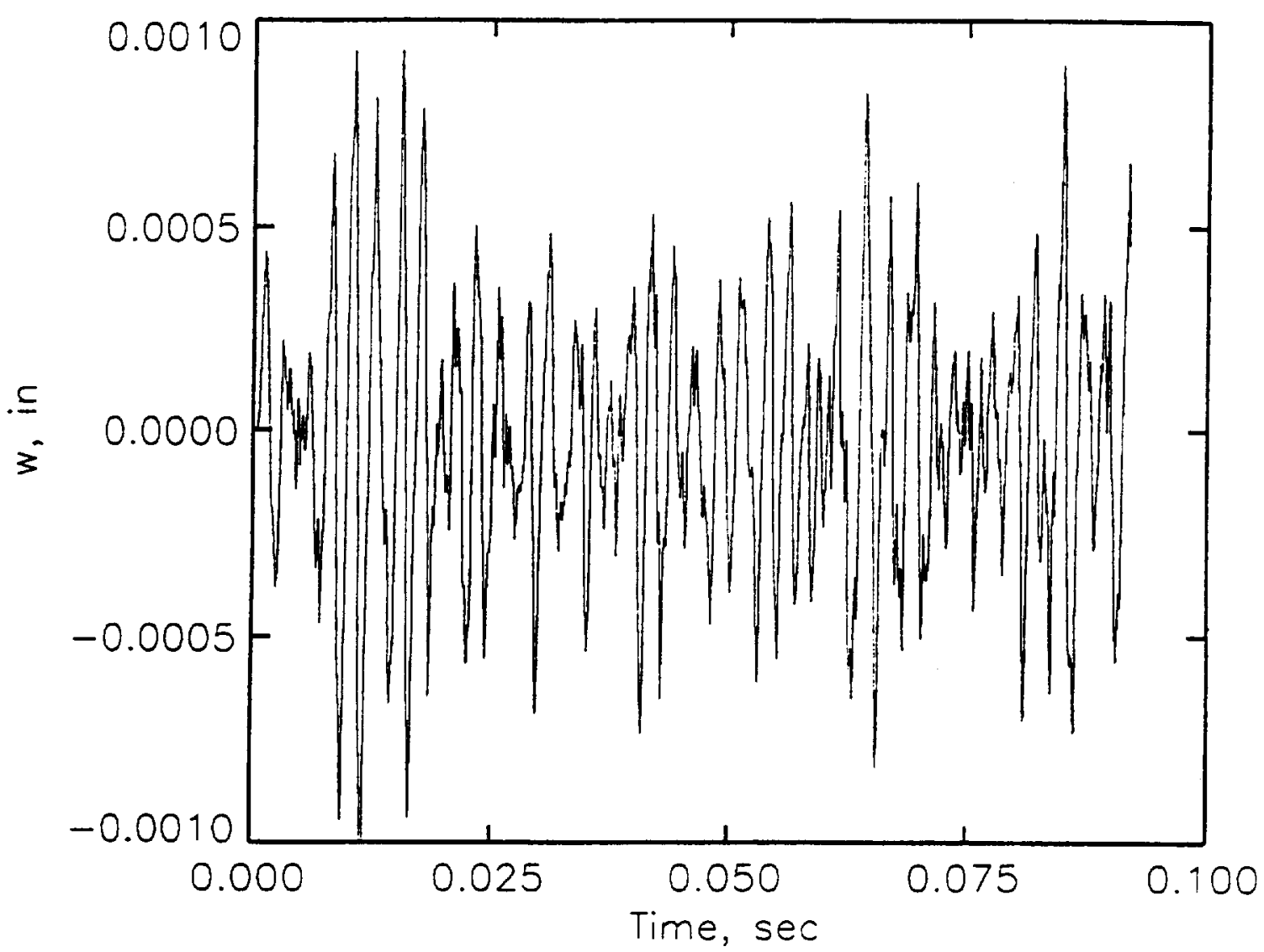

Figure 2b: Time history of the center plate displacement response. 


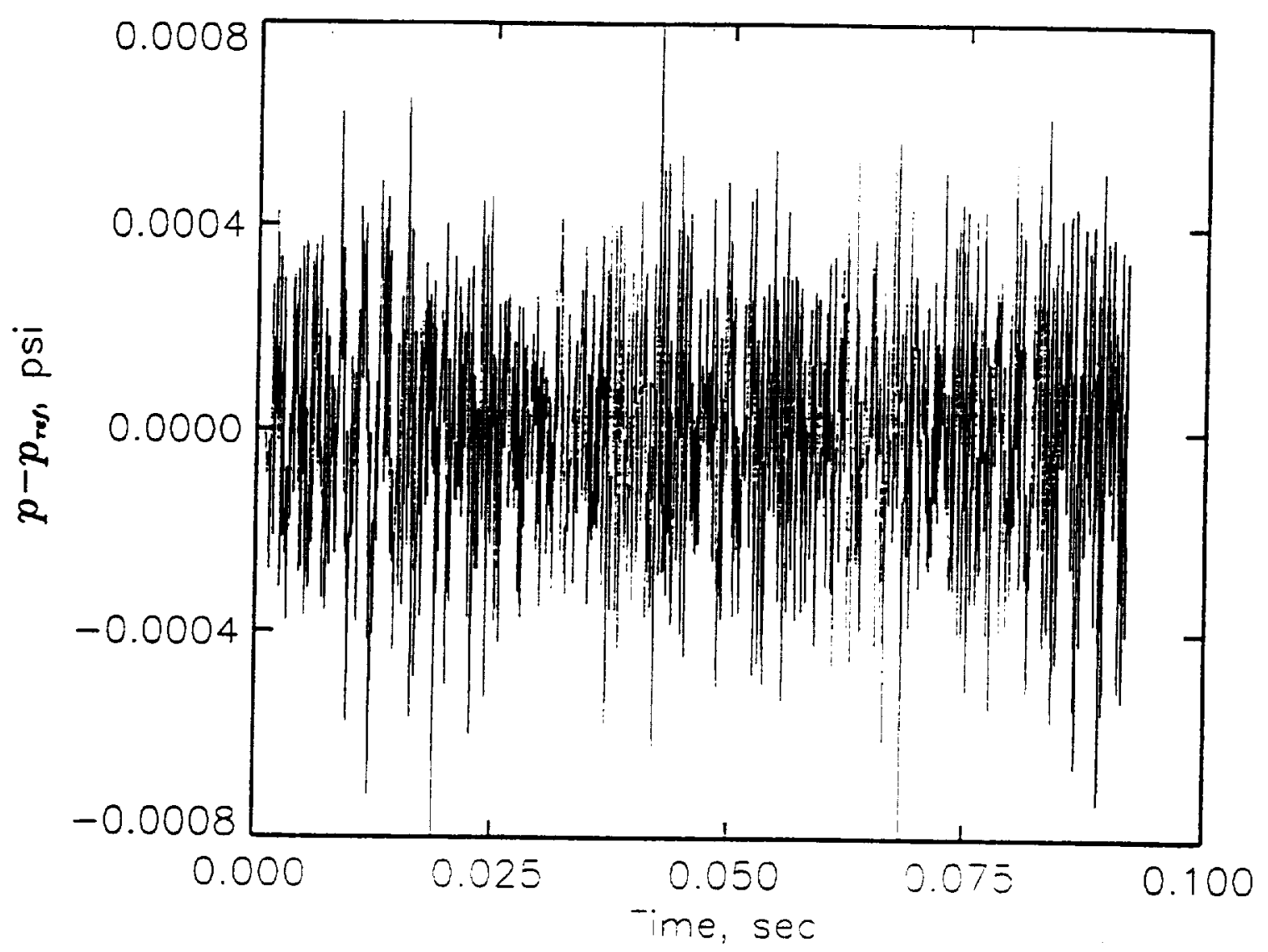

Figure 2c: Time history of the radiated pressure one foot away from the plate center. 


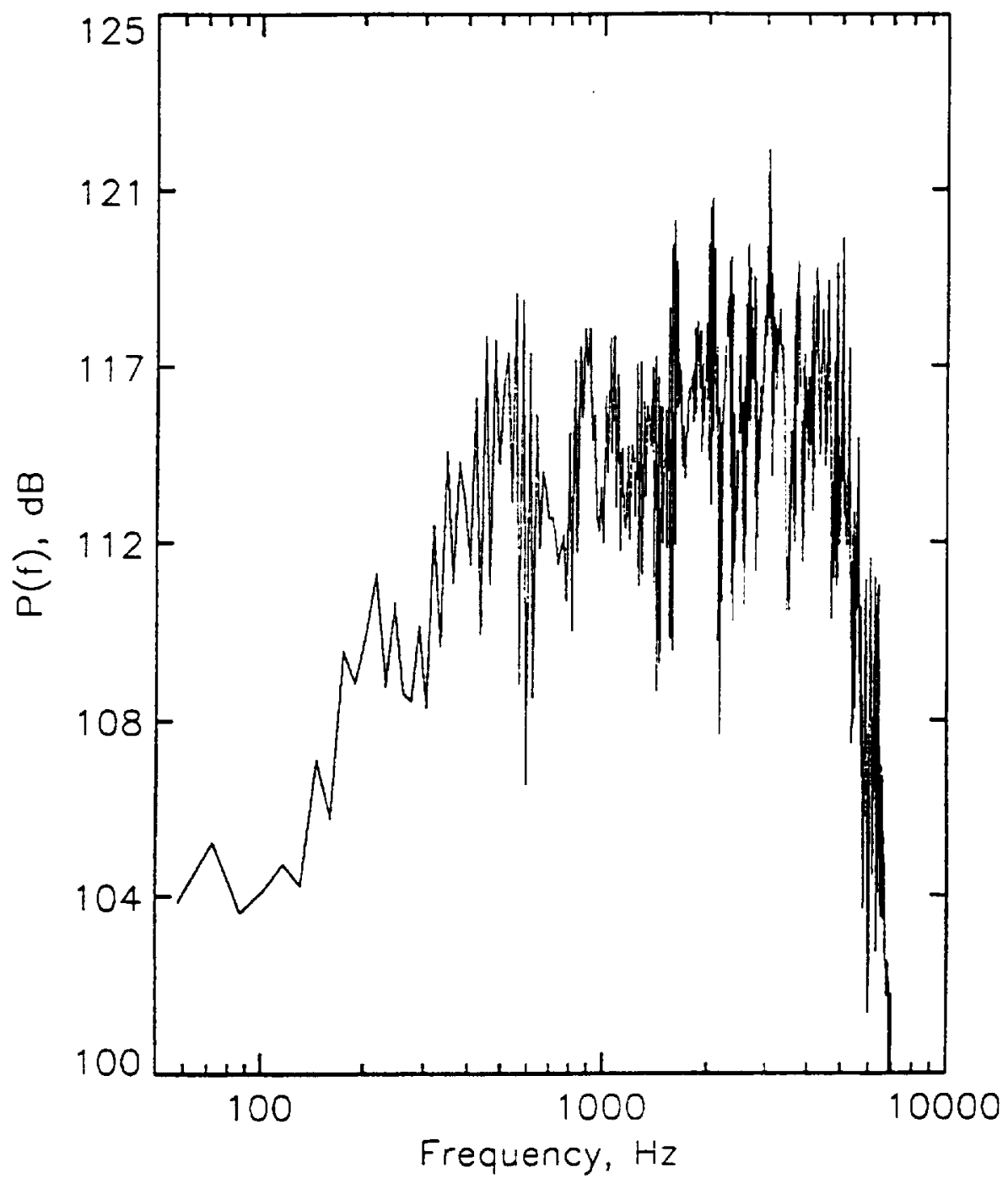

Figure 3a: Power Spectral Density (PSD) of the turbulent boundary layer surface pressure at the center of the plate. 


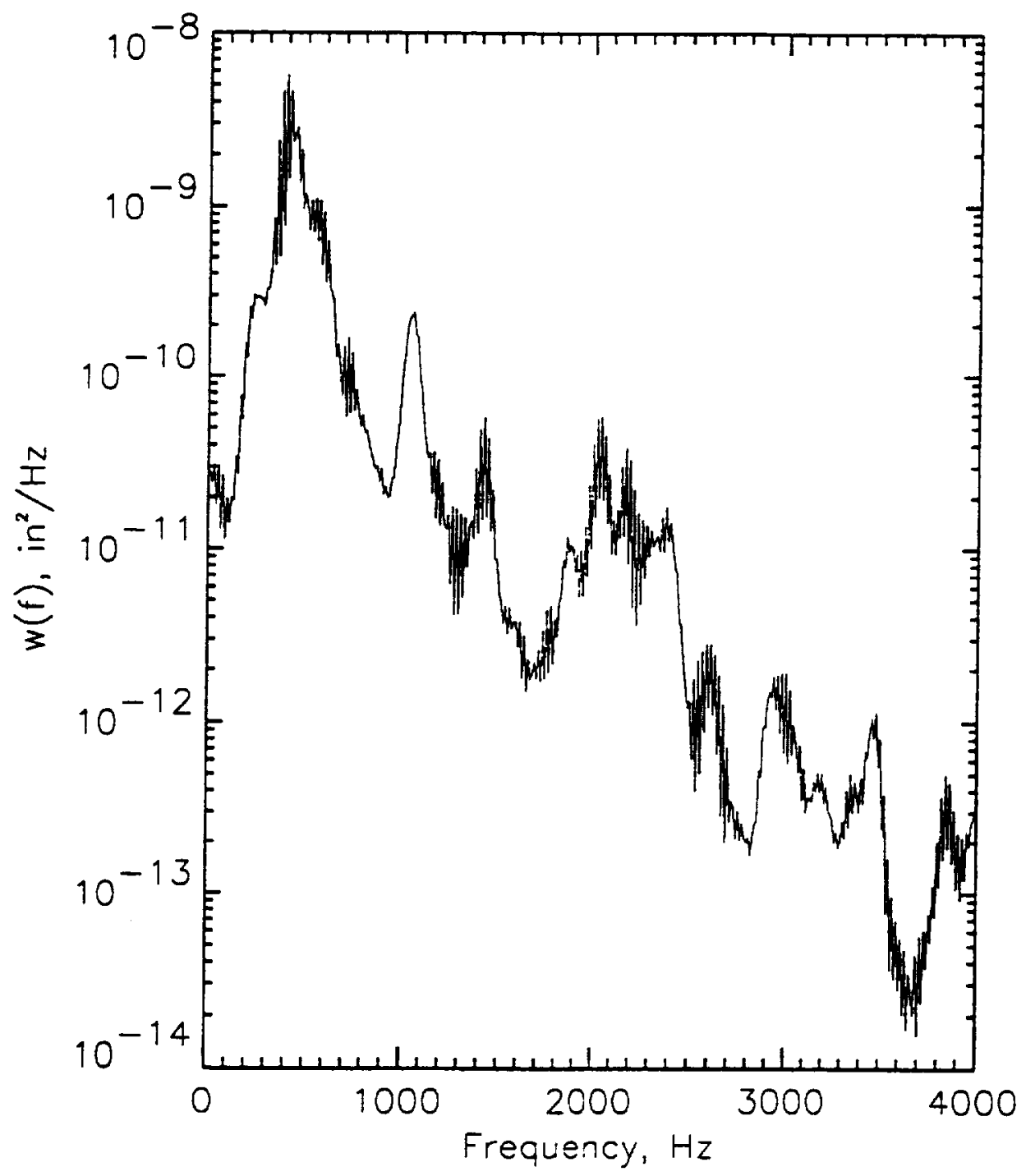

Figure 3b: Power Spectral Density (PSD) of the center plate displacement response. 


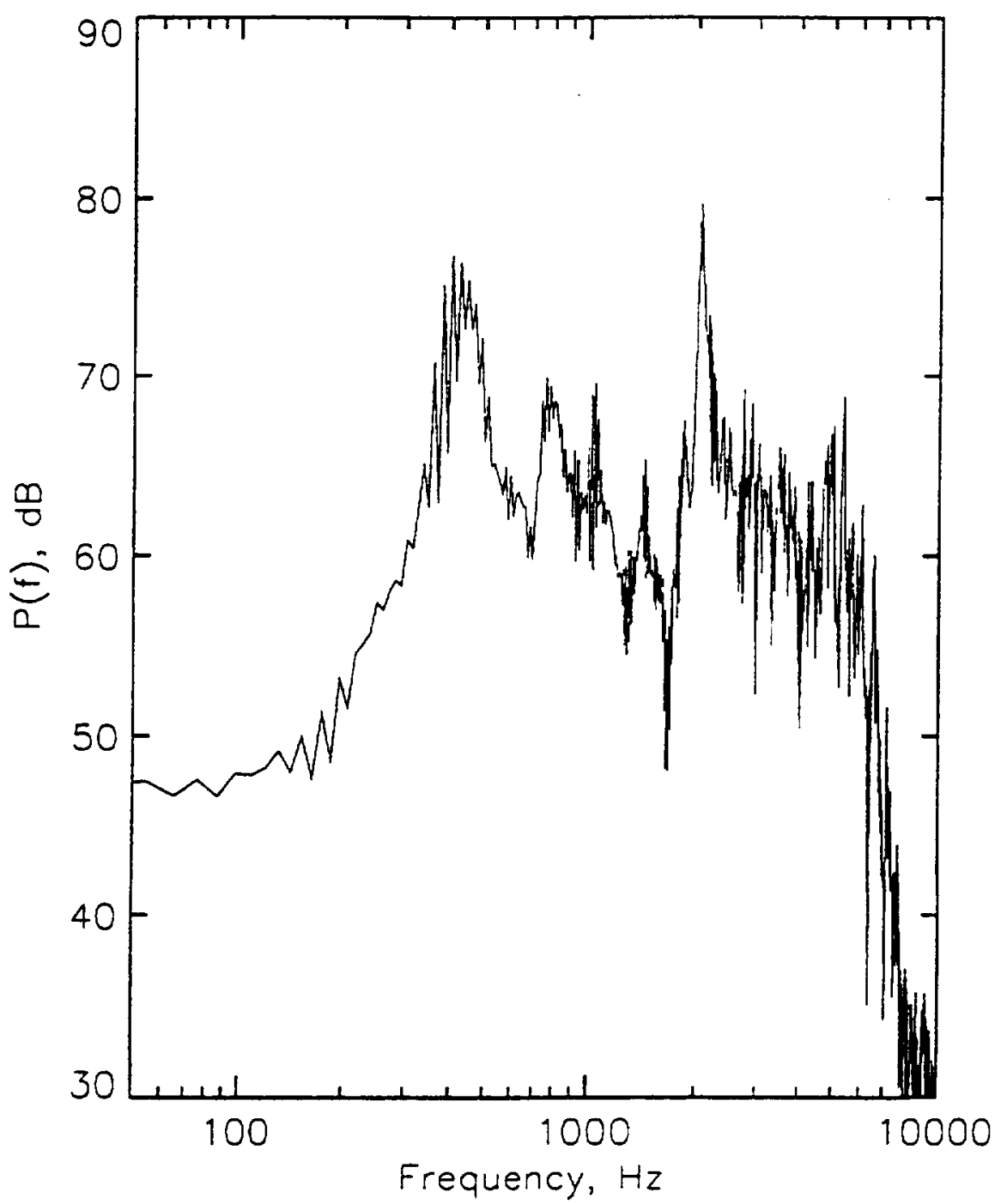

Figure 3c: Power Spectral Density (PSD) of the radiated pressure one foot away from the plate center. 


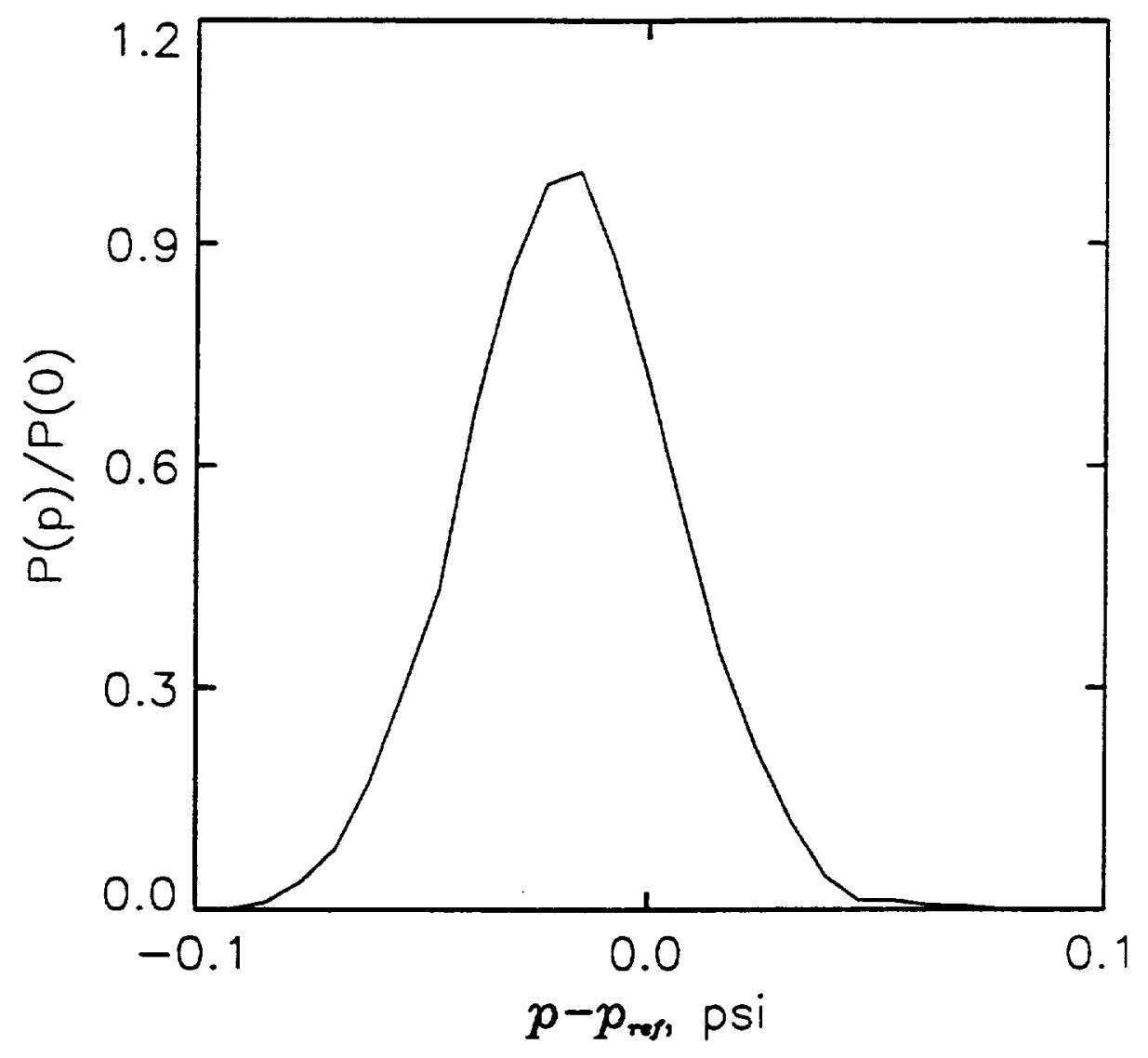

Figure 4a: Probability distribution of the turbulent boundary layer surface pressure at the center of the plate. 


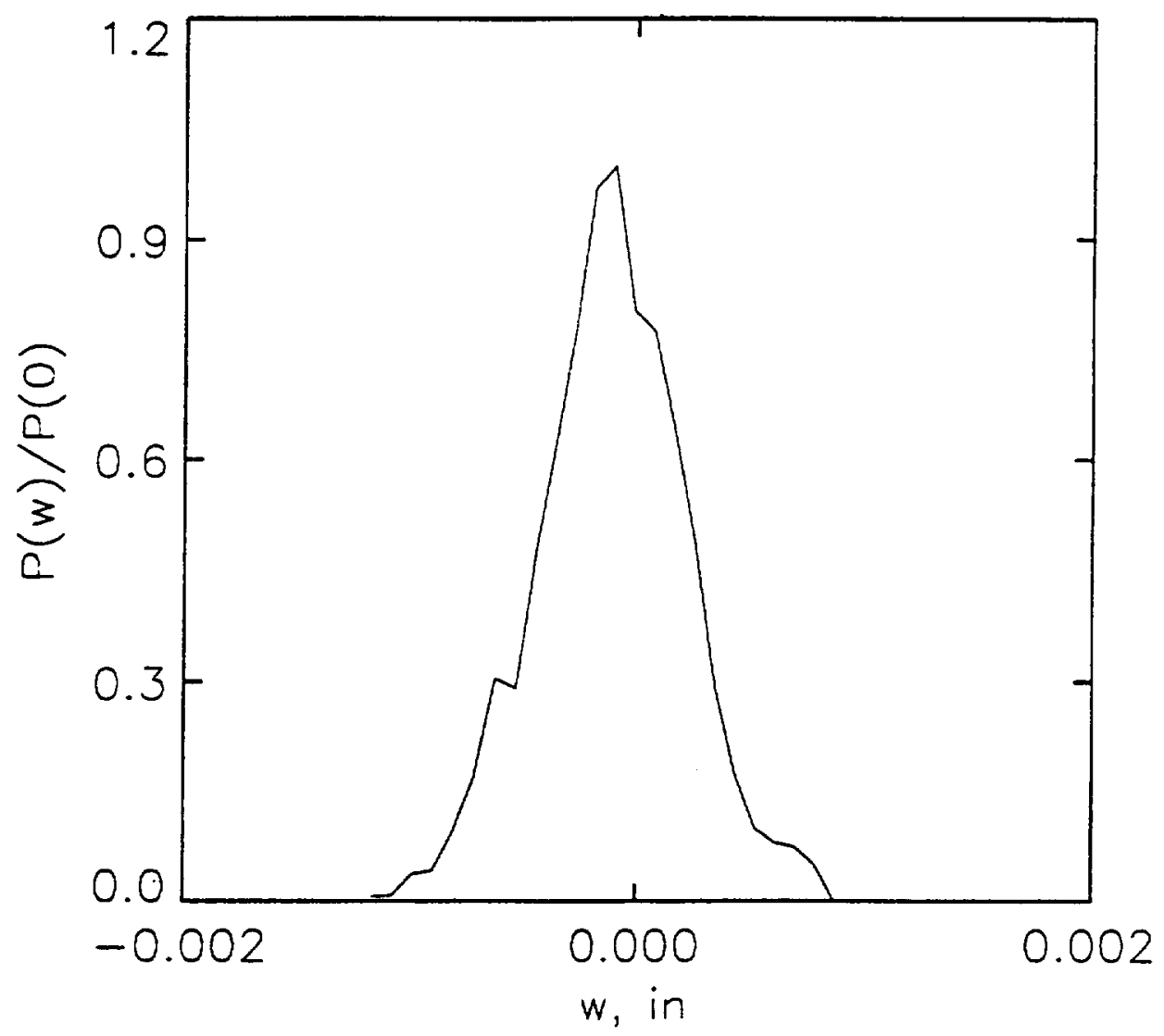

Figure 4b: Probability distribution of the center plate displacement response. 


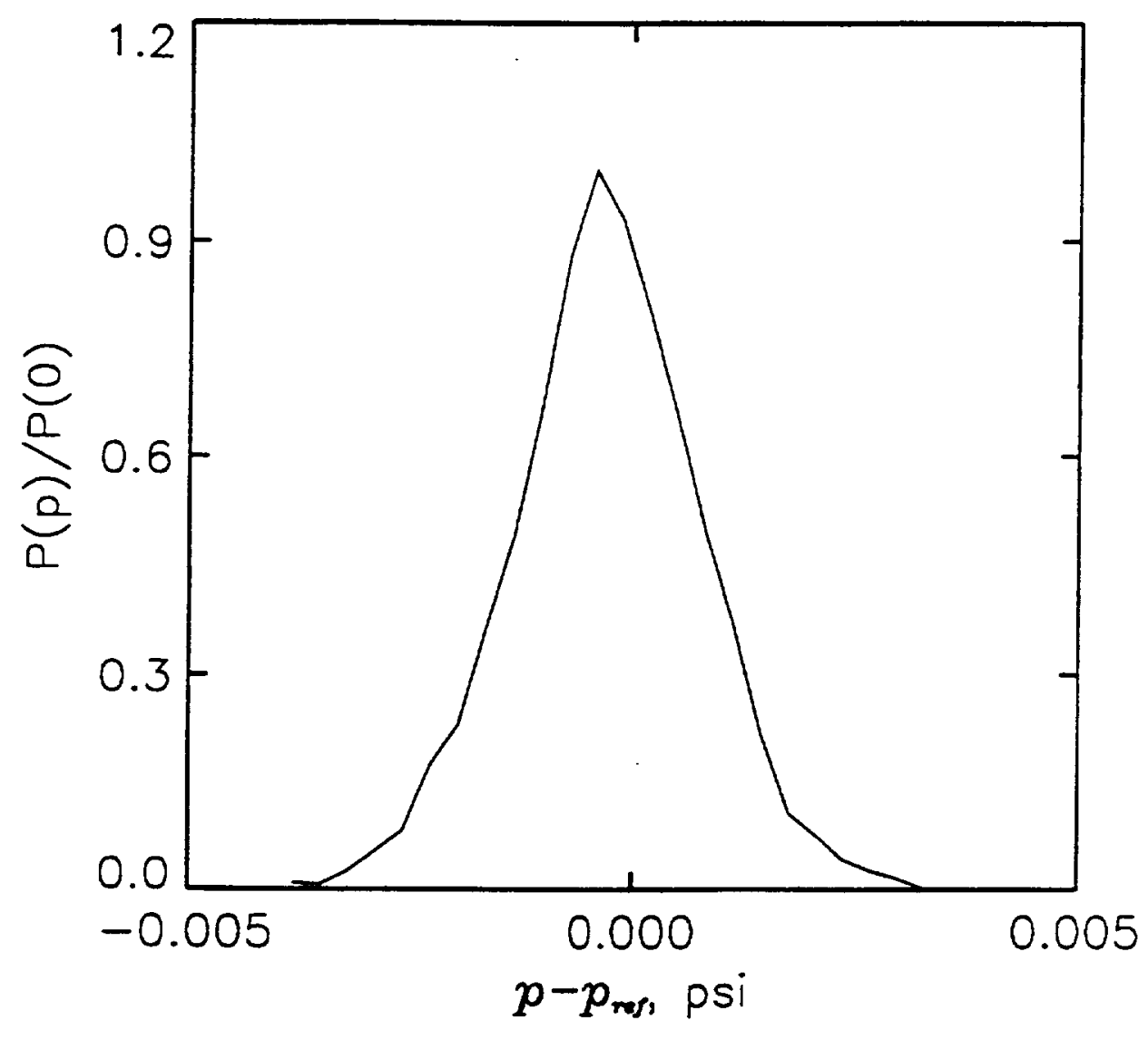

Figure 4c: Probability distribution of the radiated pressure one foot away from the plate center. 

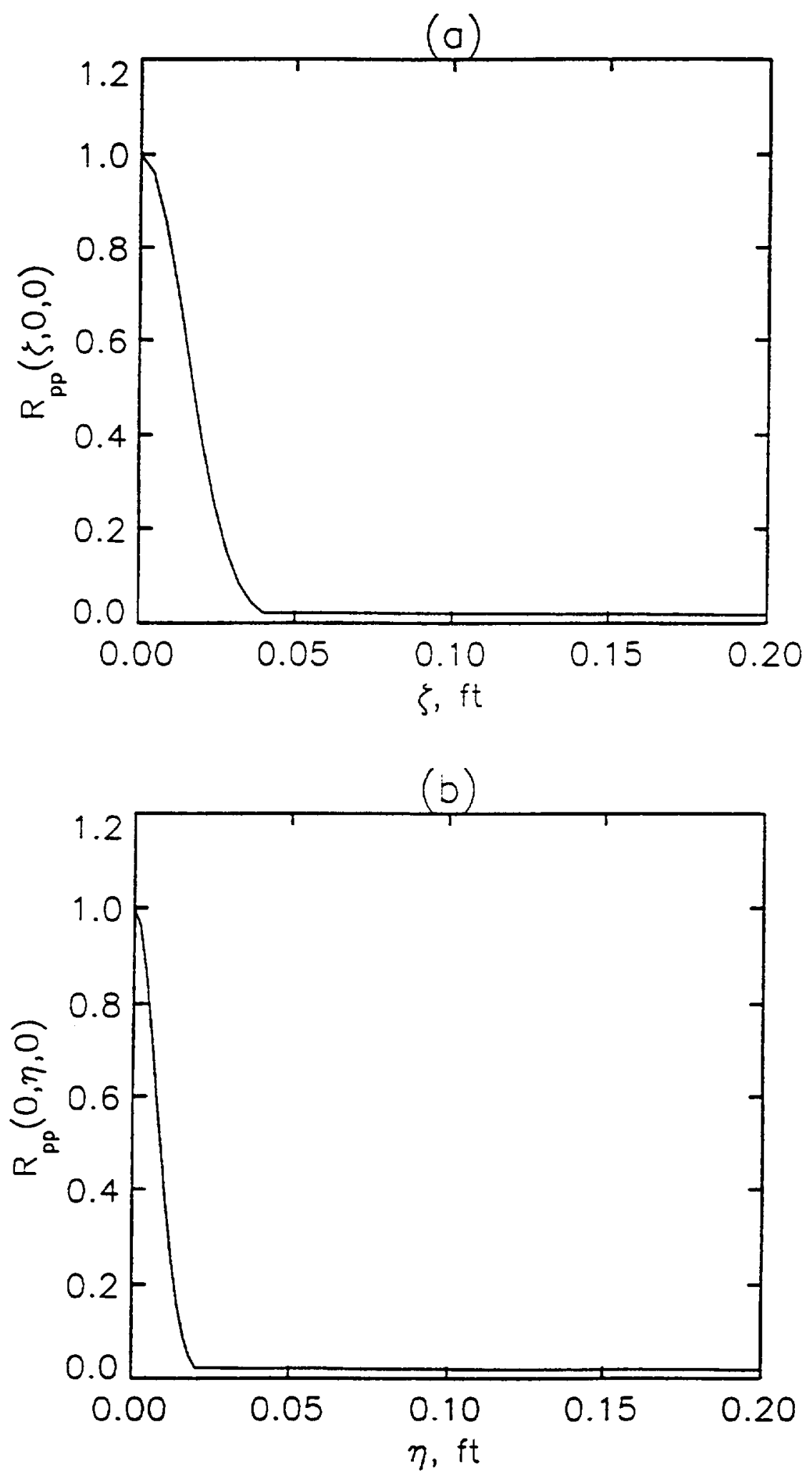

Figure 5: Two-point correlations of the wall pressure; (a) in the streamwise direction, (b) in the spanwise direction. 


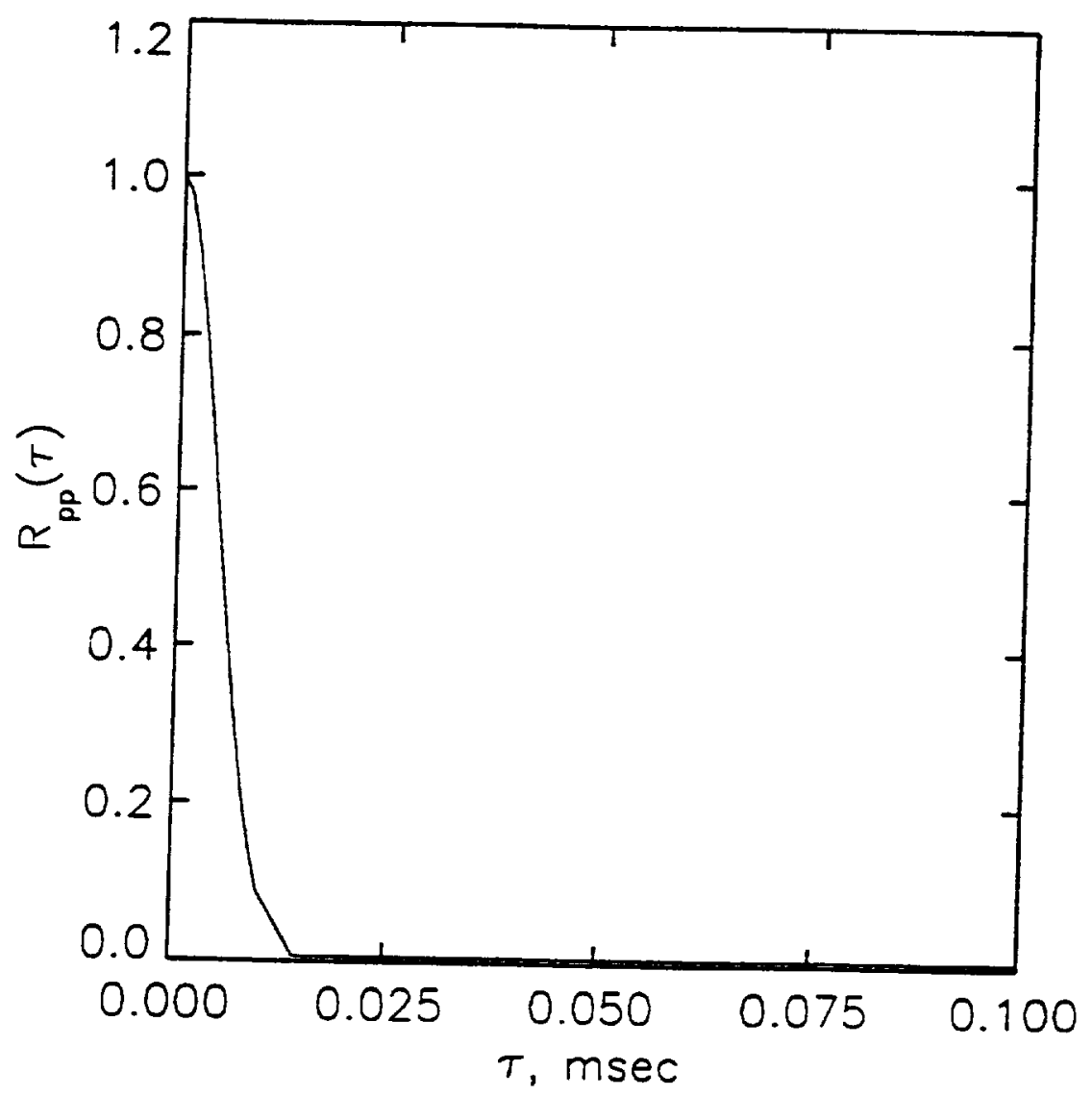

Figure 6: Auto-correlation of the wall pressure. 


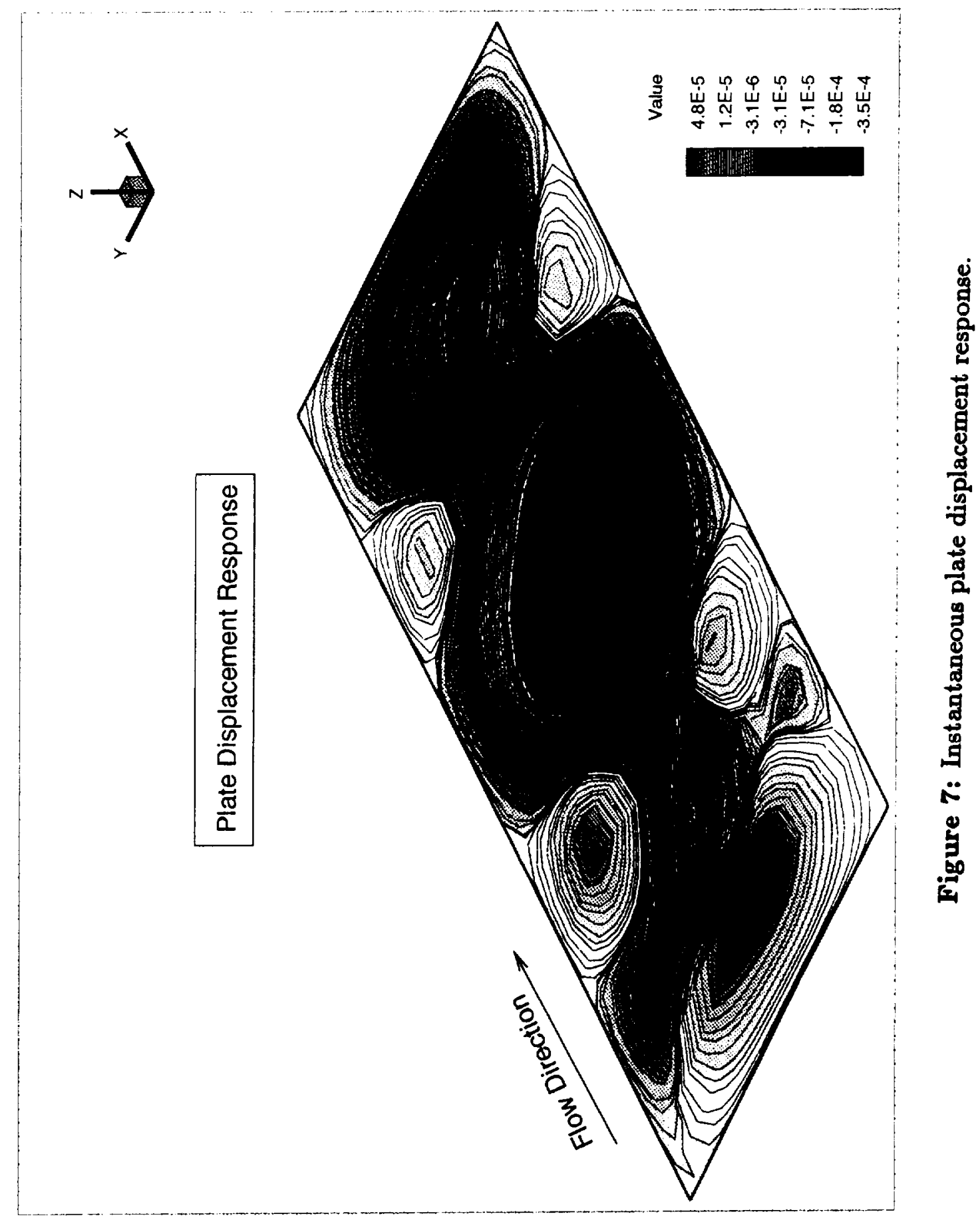




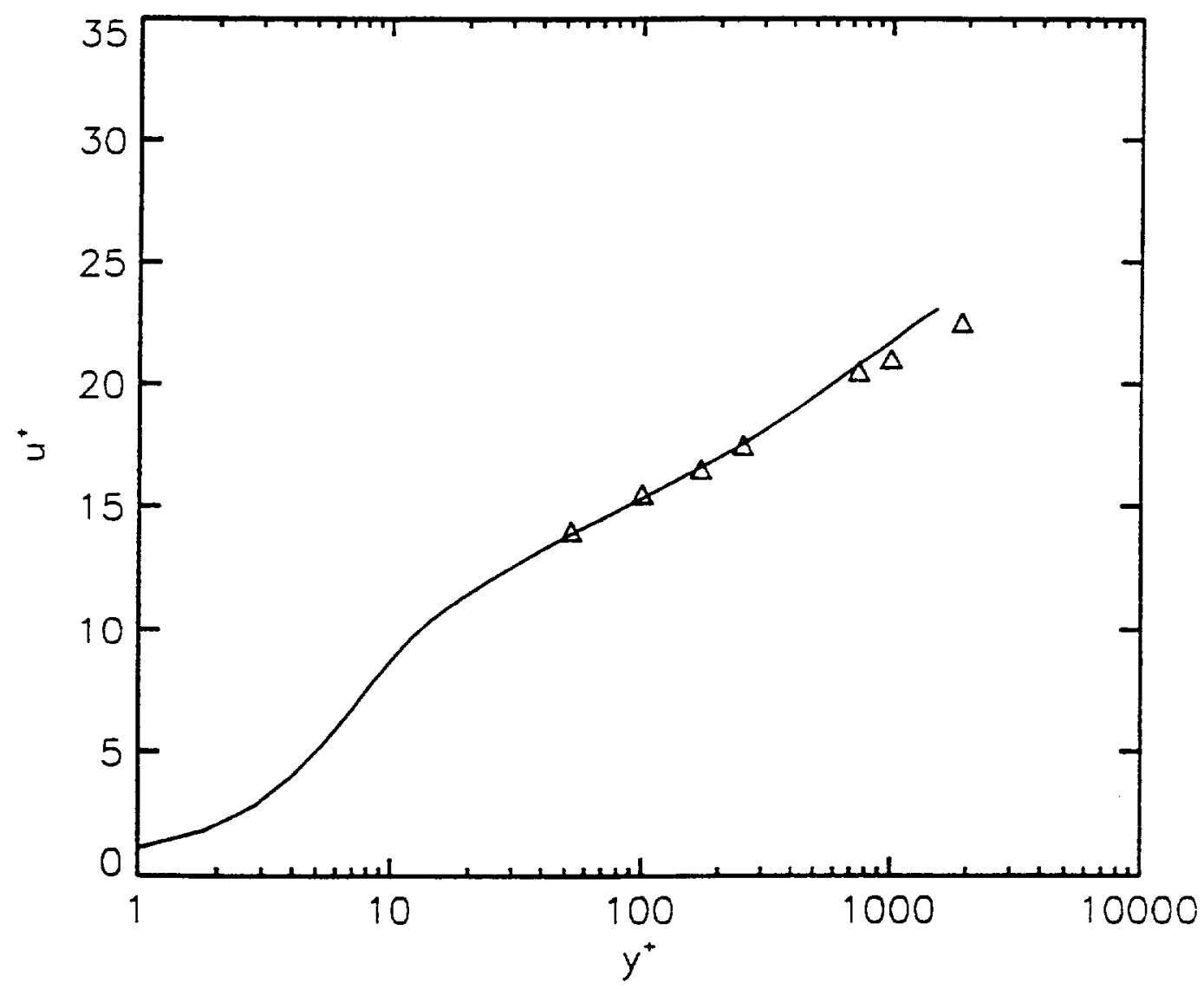

Figure 8: Mean velocity profile, $\Delta$ Experimental results (Maestrello [40]), Numerical results. 


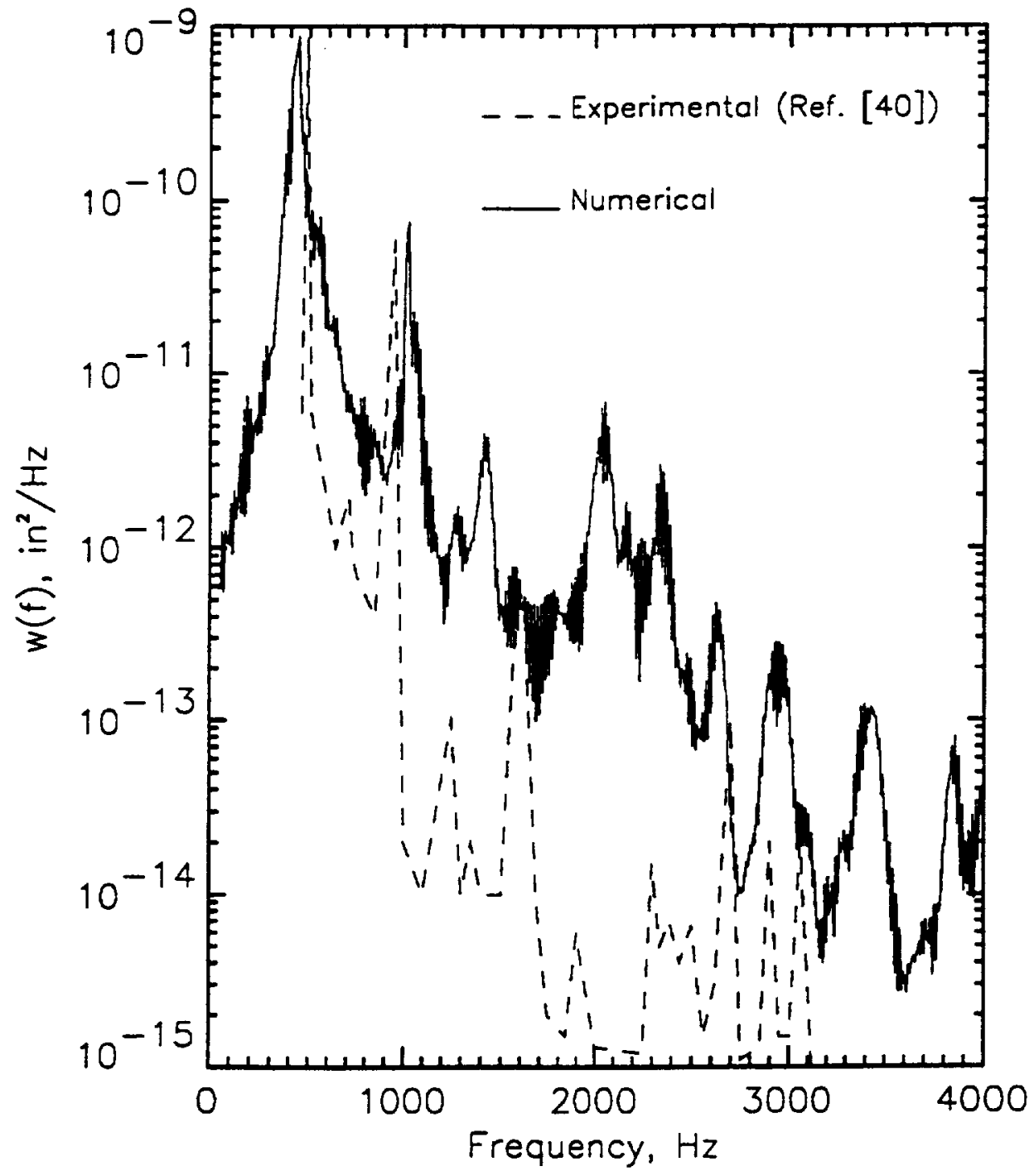

Figure 9: Comparison of the Power Spectral Density (PSD) of the center plate displacement response. 


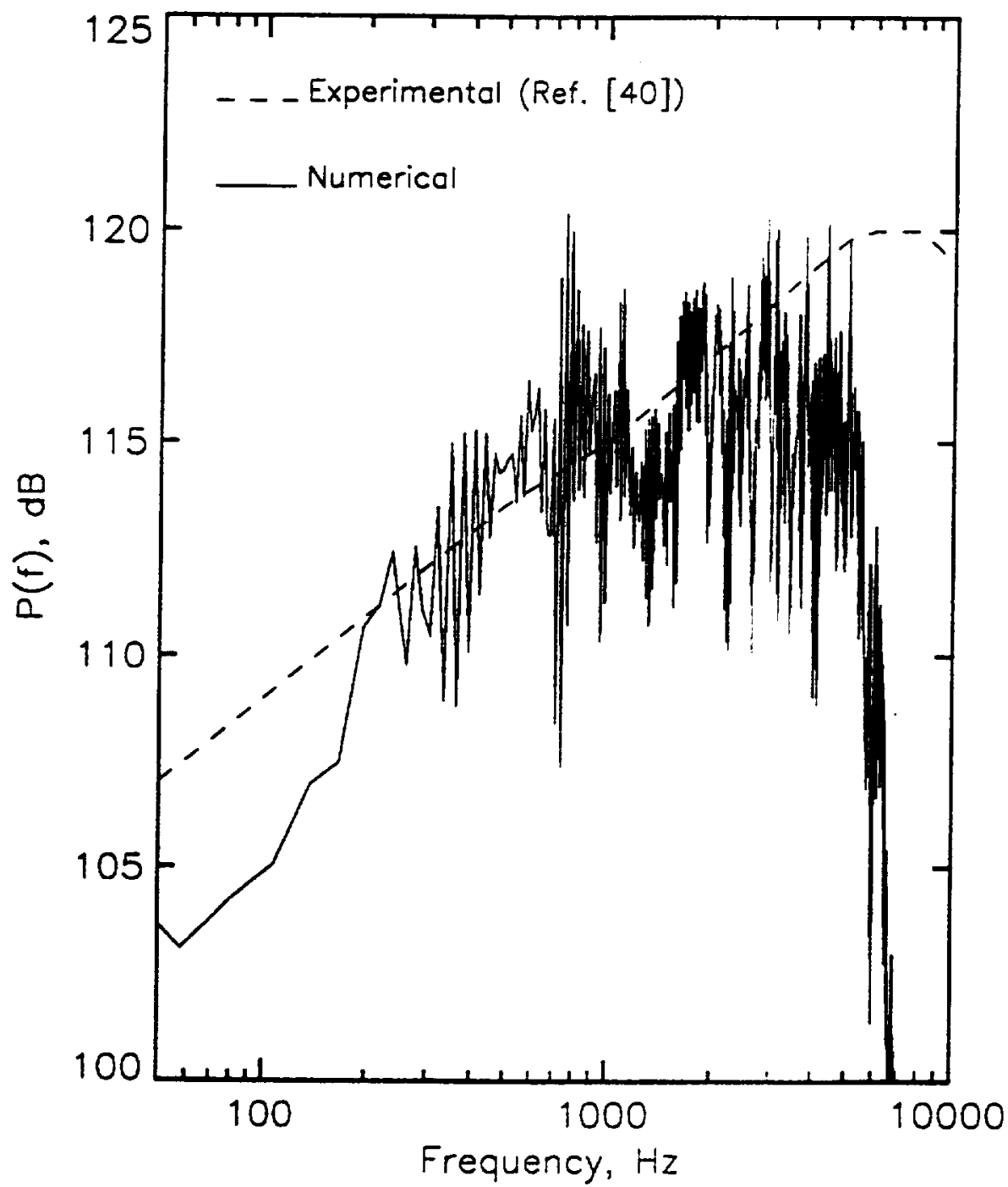

Figure 10: Comparison of the Power Spectral Density (PSD) of the turbulent boundary layer surface pressure at the center of the flexible plate. 


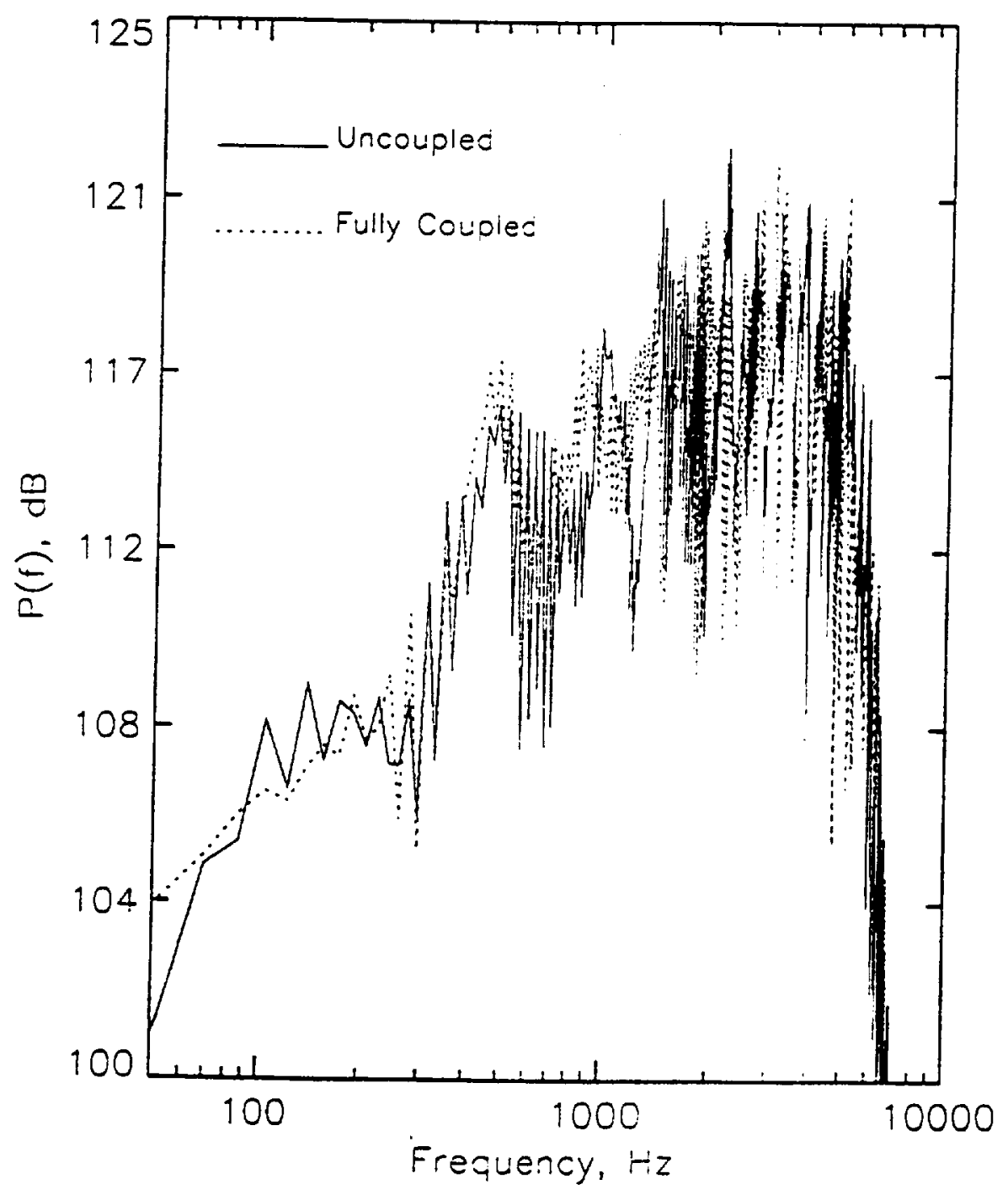

Figure 11: Comparison of the PSDs of the surface pressure at the center of the plate for the uncoupled and coupled cases. 


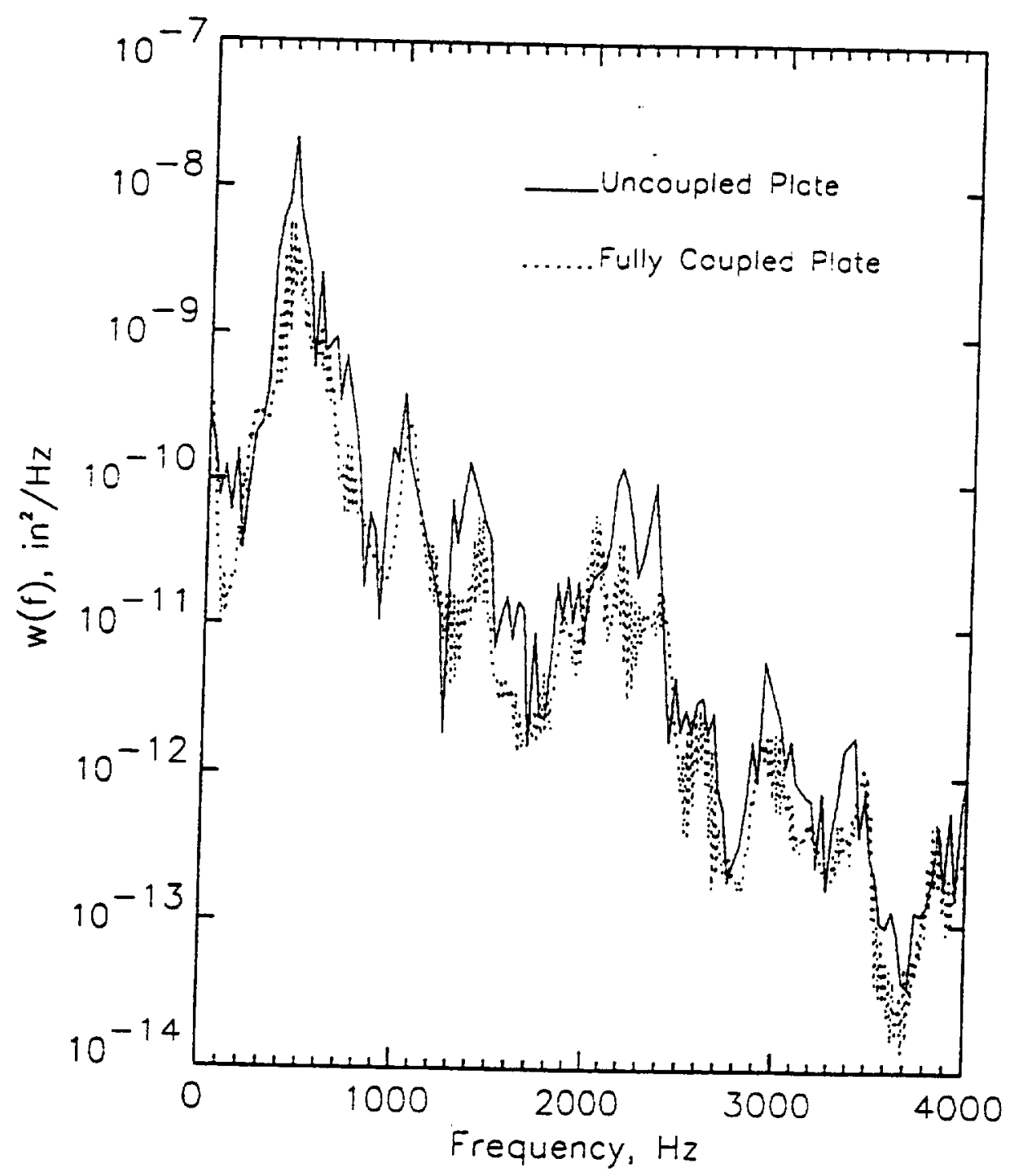

Figure 12: Comparison of the PSDs of the center plate displacement for the uncoupled and coupled cases. 


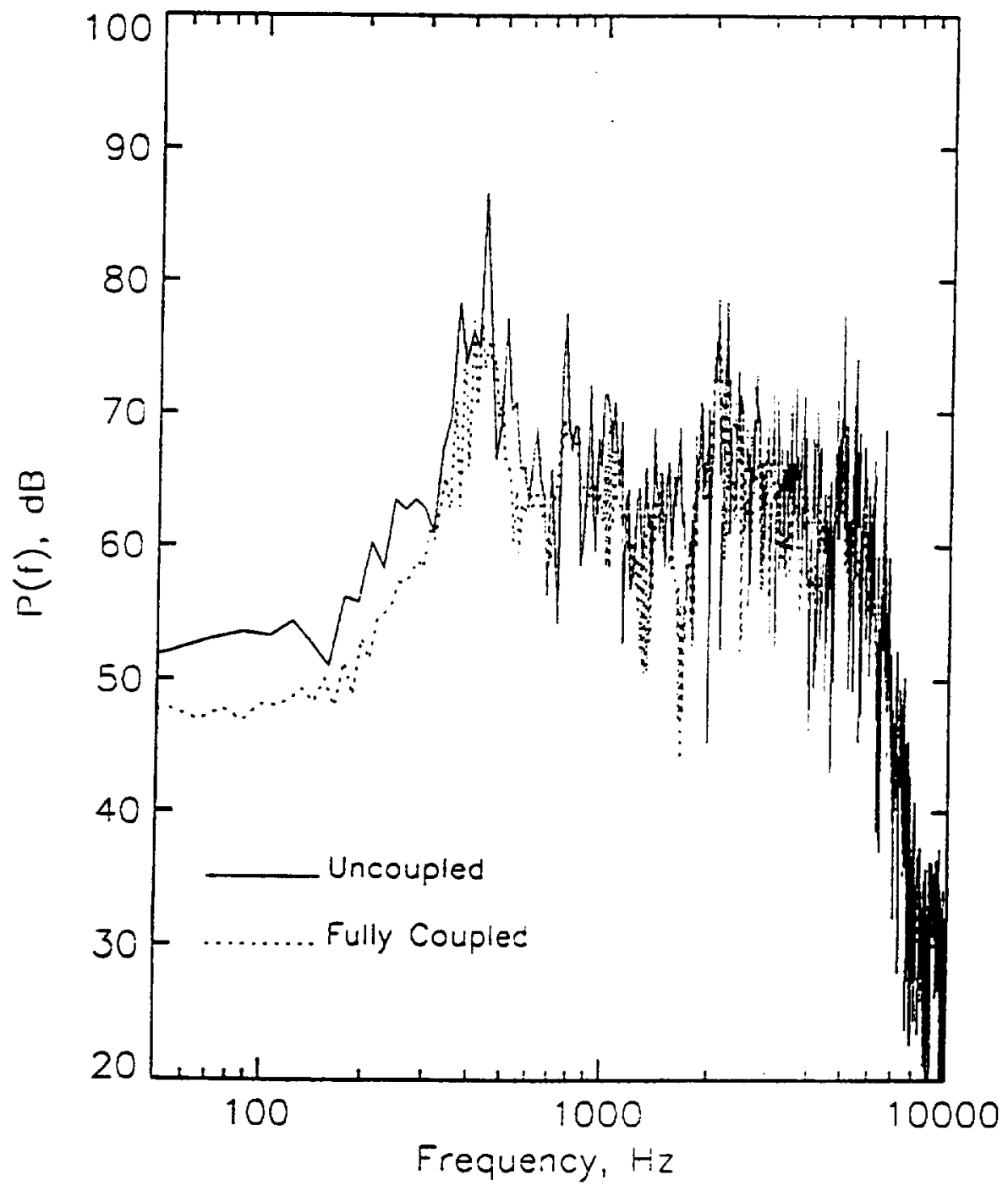

Figure 13: Comparison of the PSDs of the radiated pressure one foot away from the plate for the uncoupled and coupled cases. 


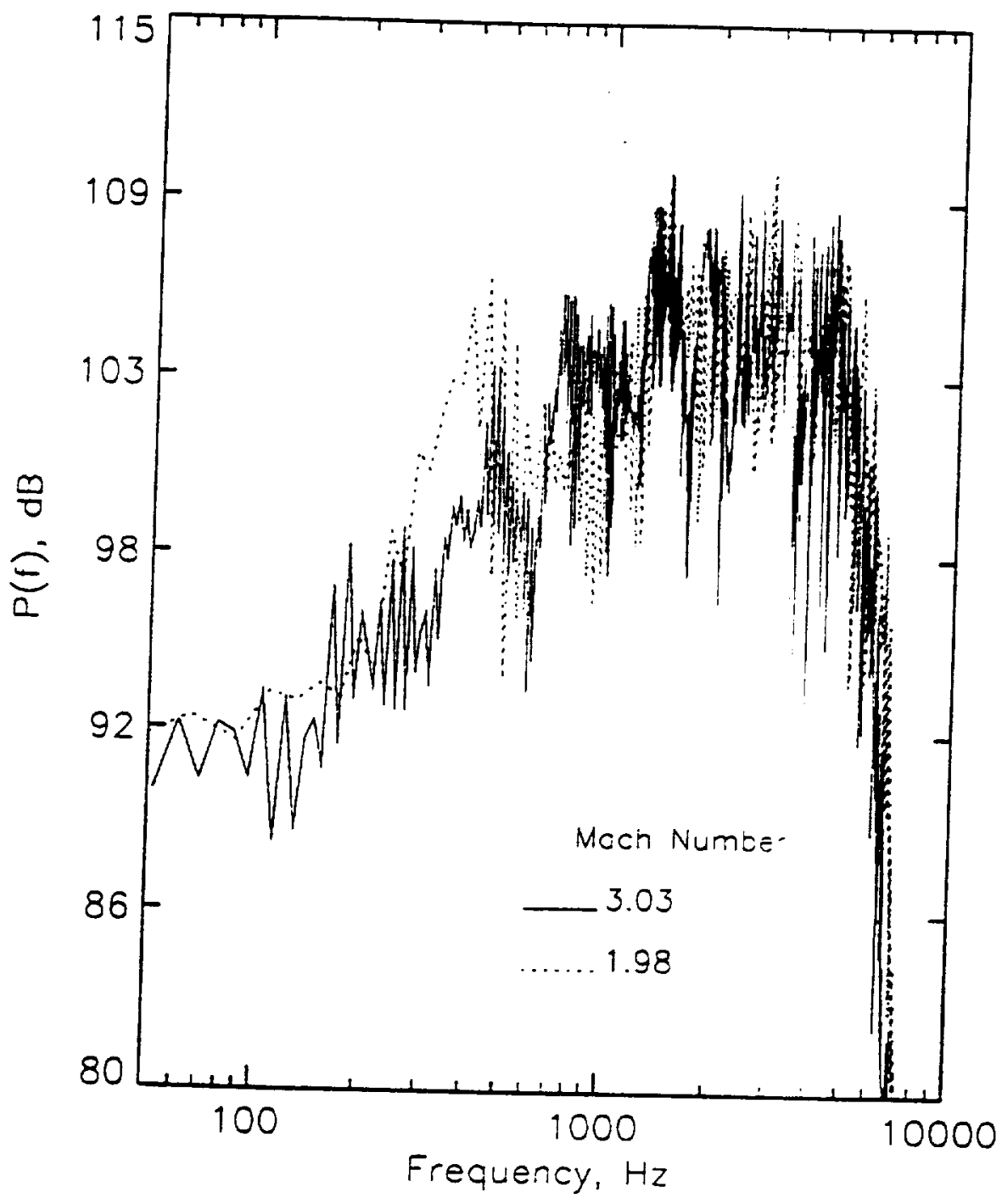

Figure 14: Comparison of the PSDs of the surface pressure at the center of the plate for two different Mach numbers. 


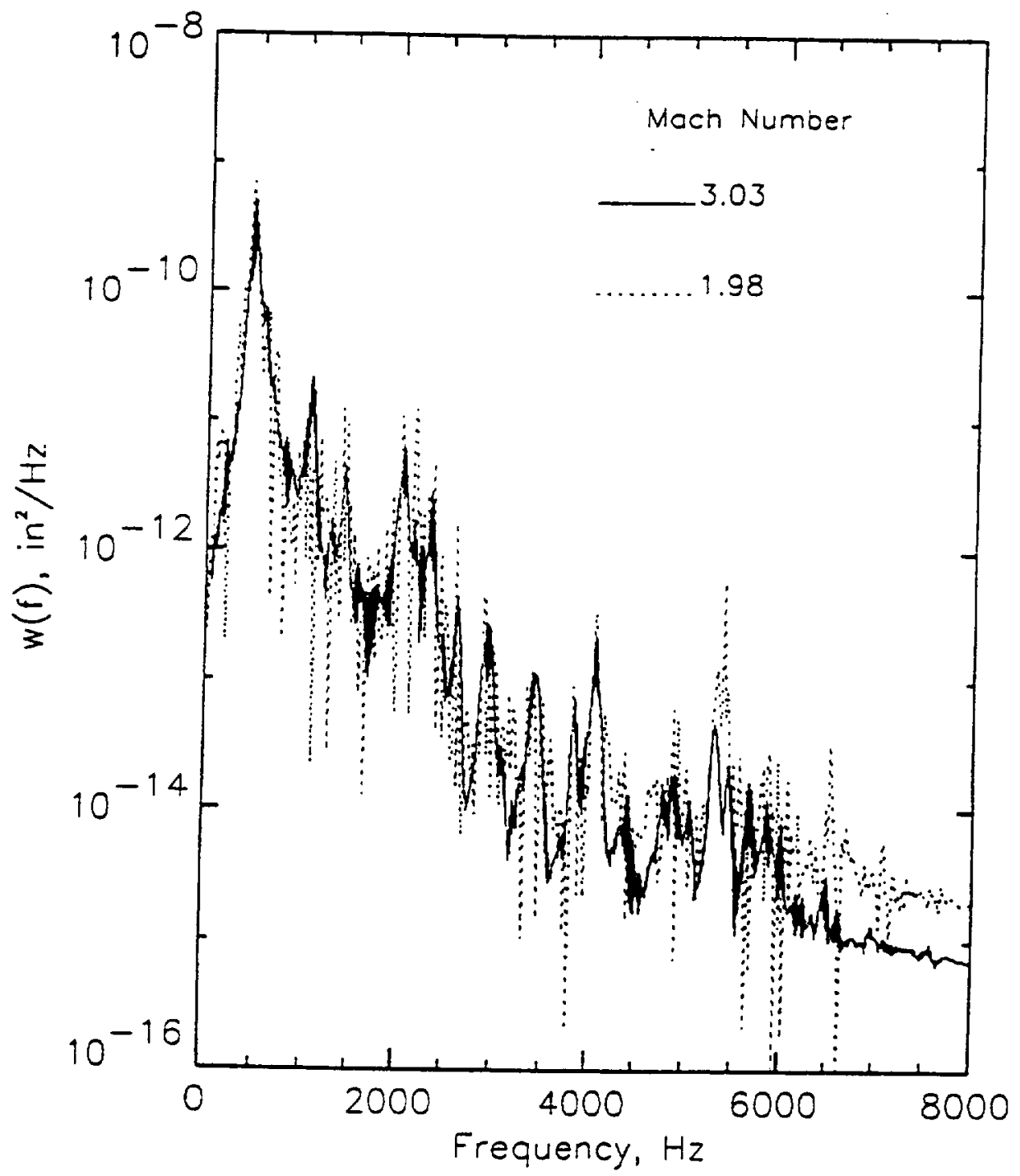

Figure 15: Comparison of the PSDs of the center plate displacement for two different Mach numbers. 


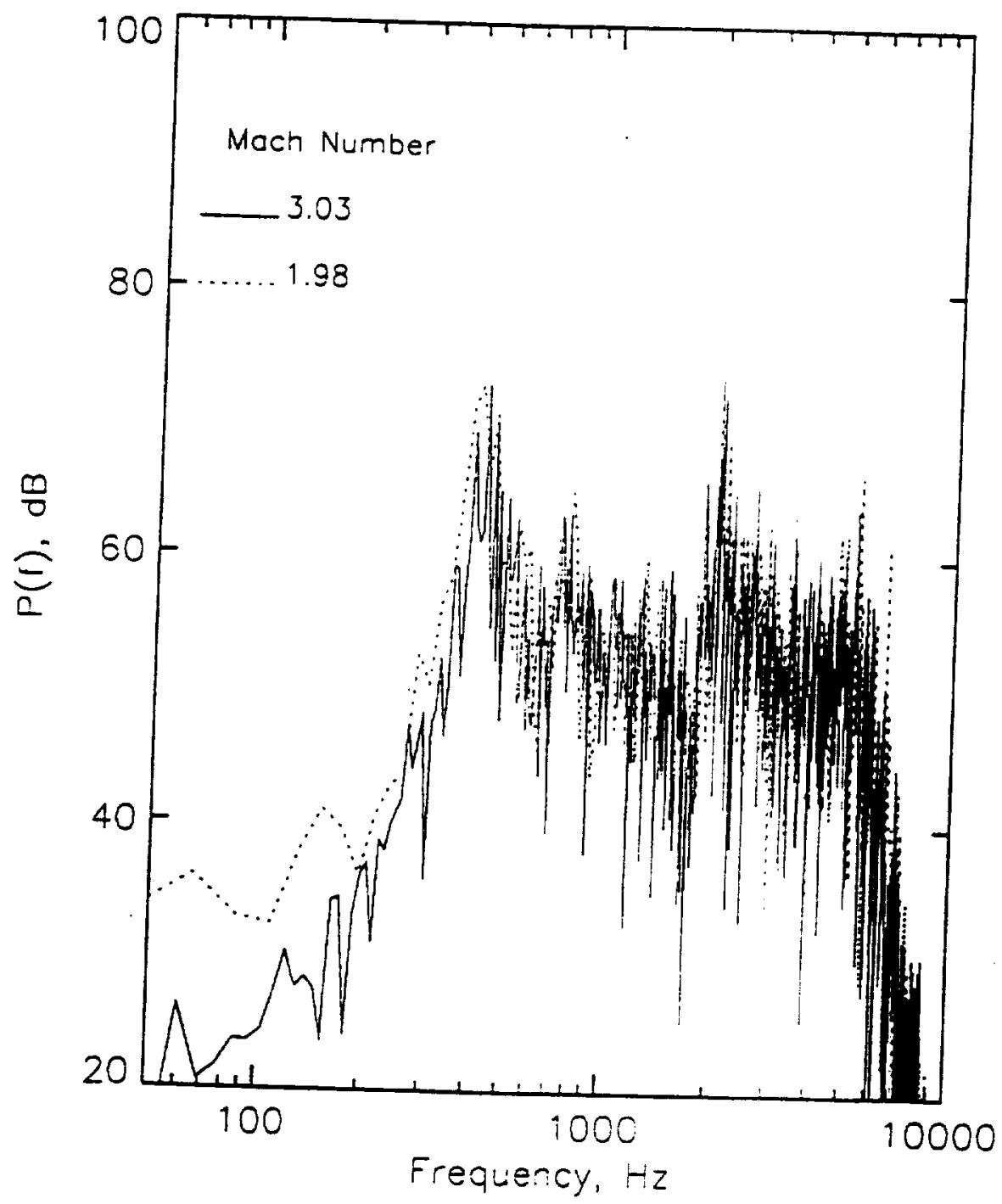

Figure 16: Comparison of the PSDs of the radiated pressure one foot away from the plate for two different Mach numbers. 


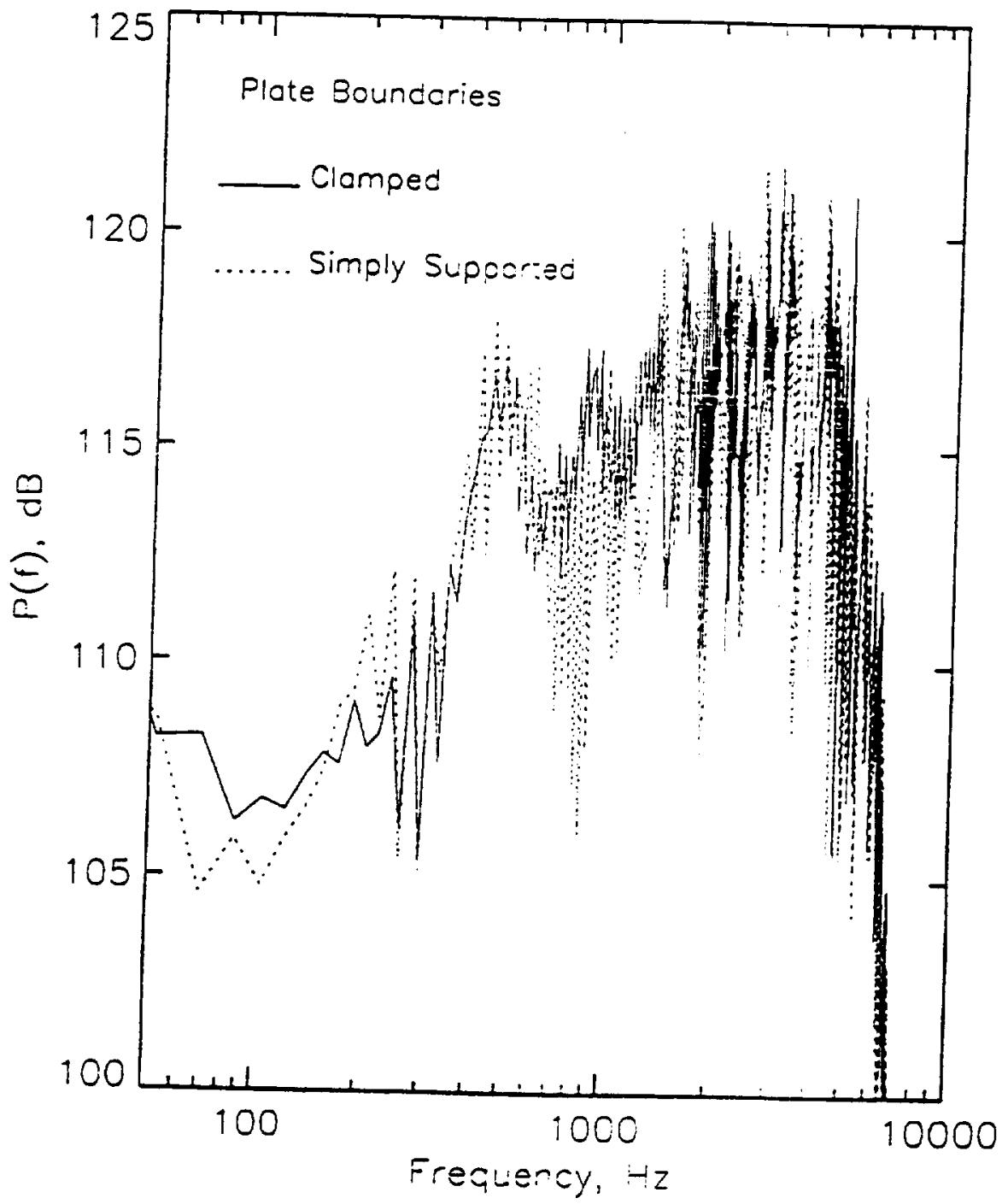

Figure 17: Comparison of the PSDs of the surface pressure at the center of the plate for two edge conditions of the plate. 


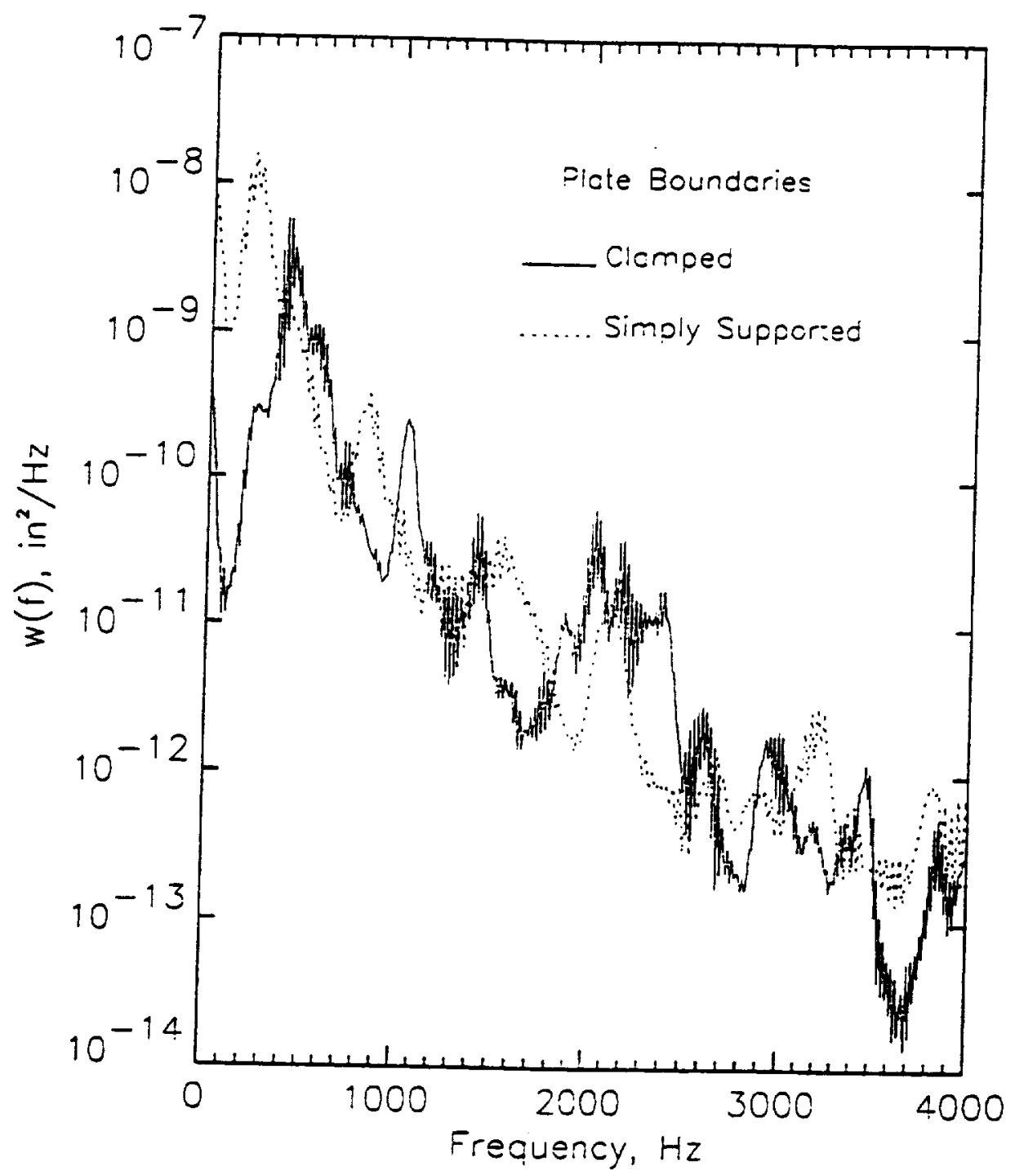

Figure 18: Comparison of the PSDs of the center plate displacement for two edge conditions of the plate. 


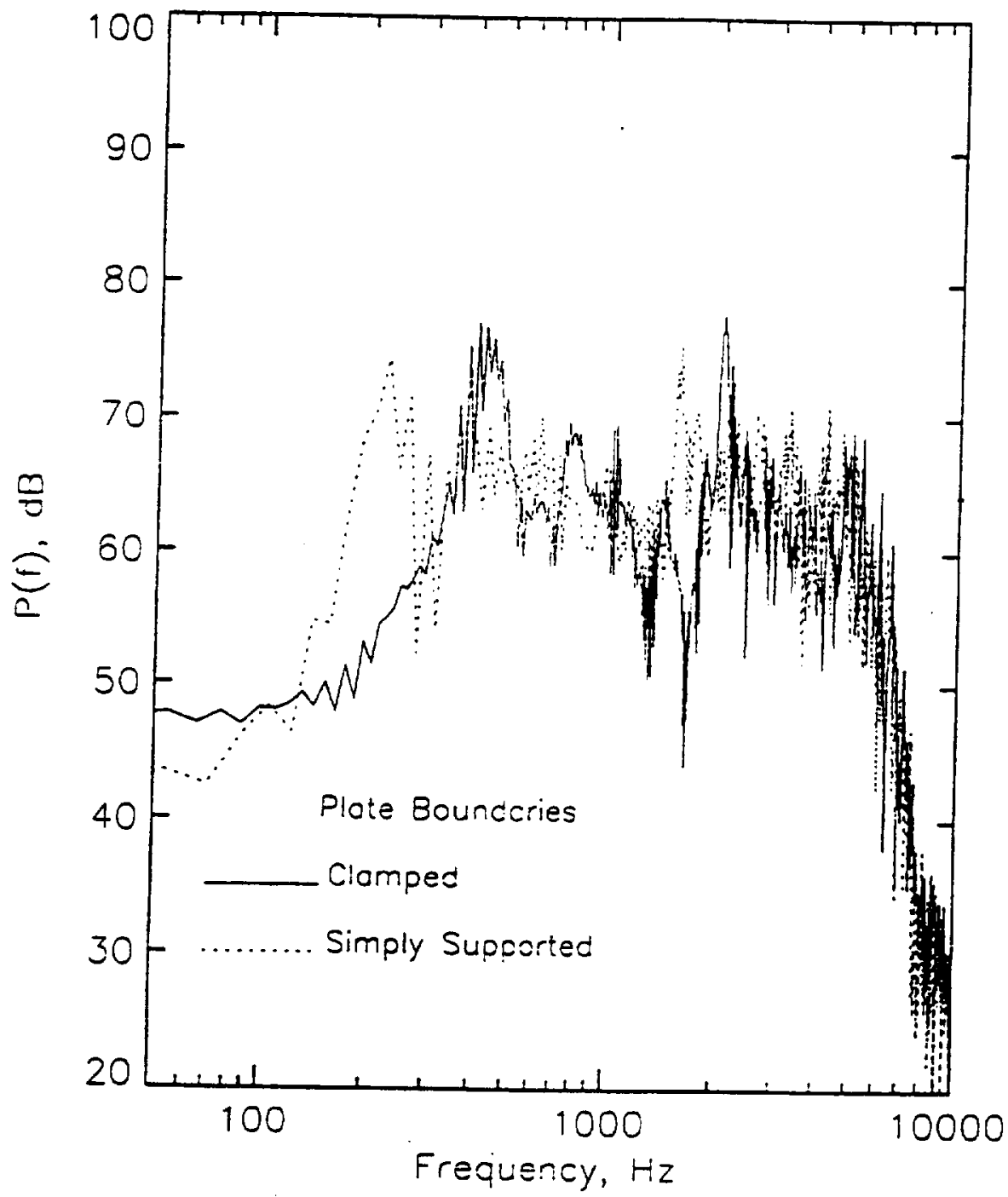

Figure 19: Comparison of the PSDs of the radiated pressure one foot away from the plate for two edge conditions of the plate. 


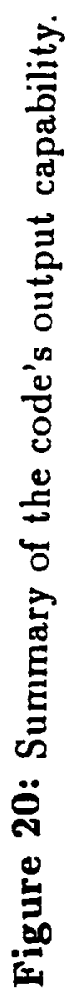

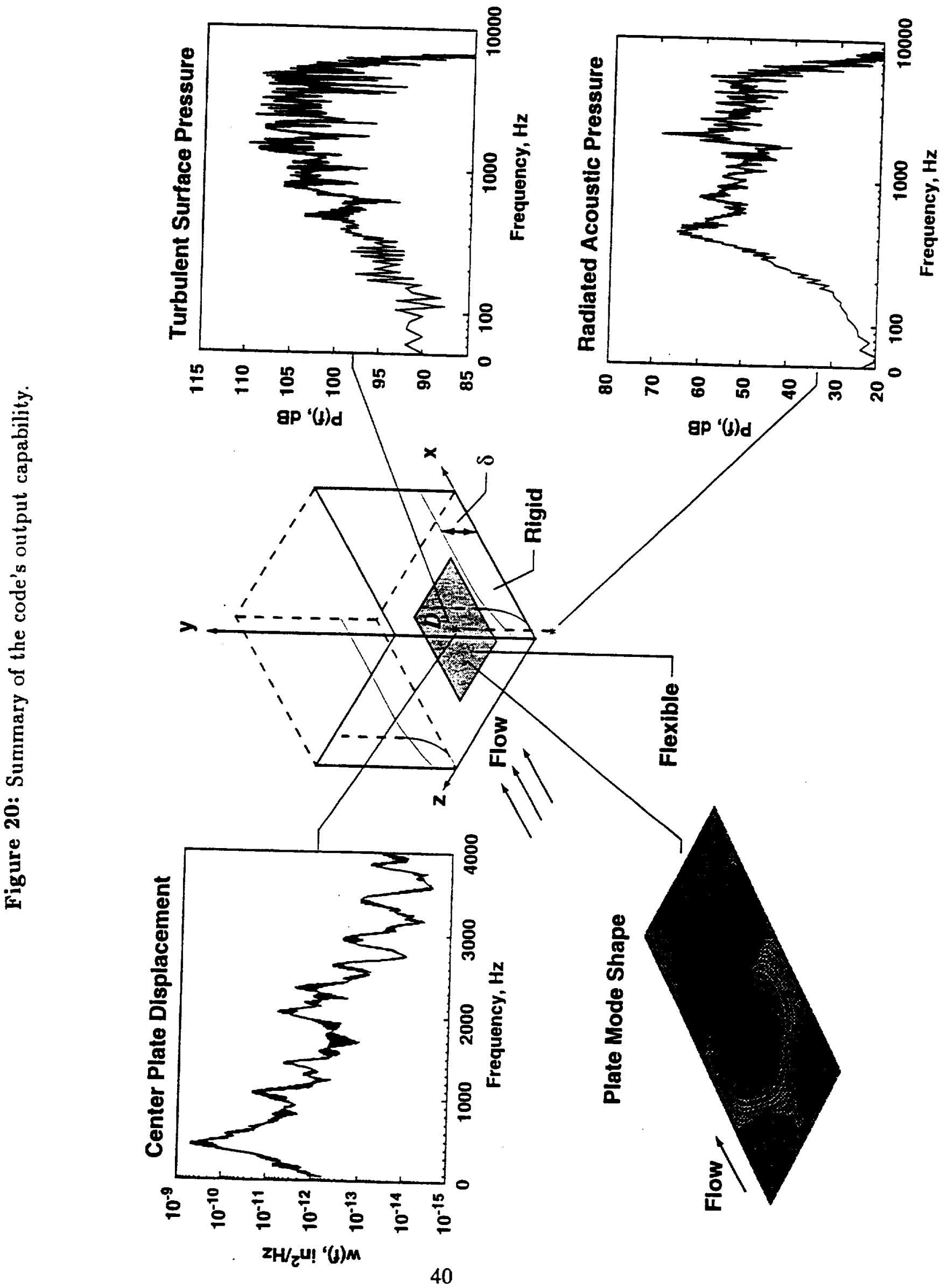




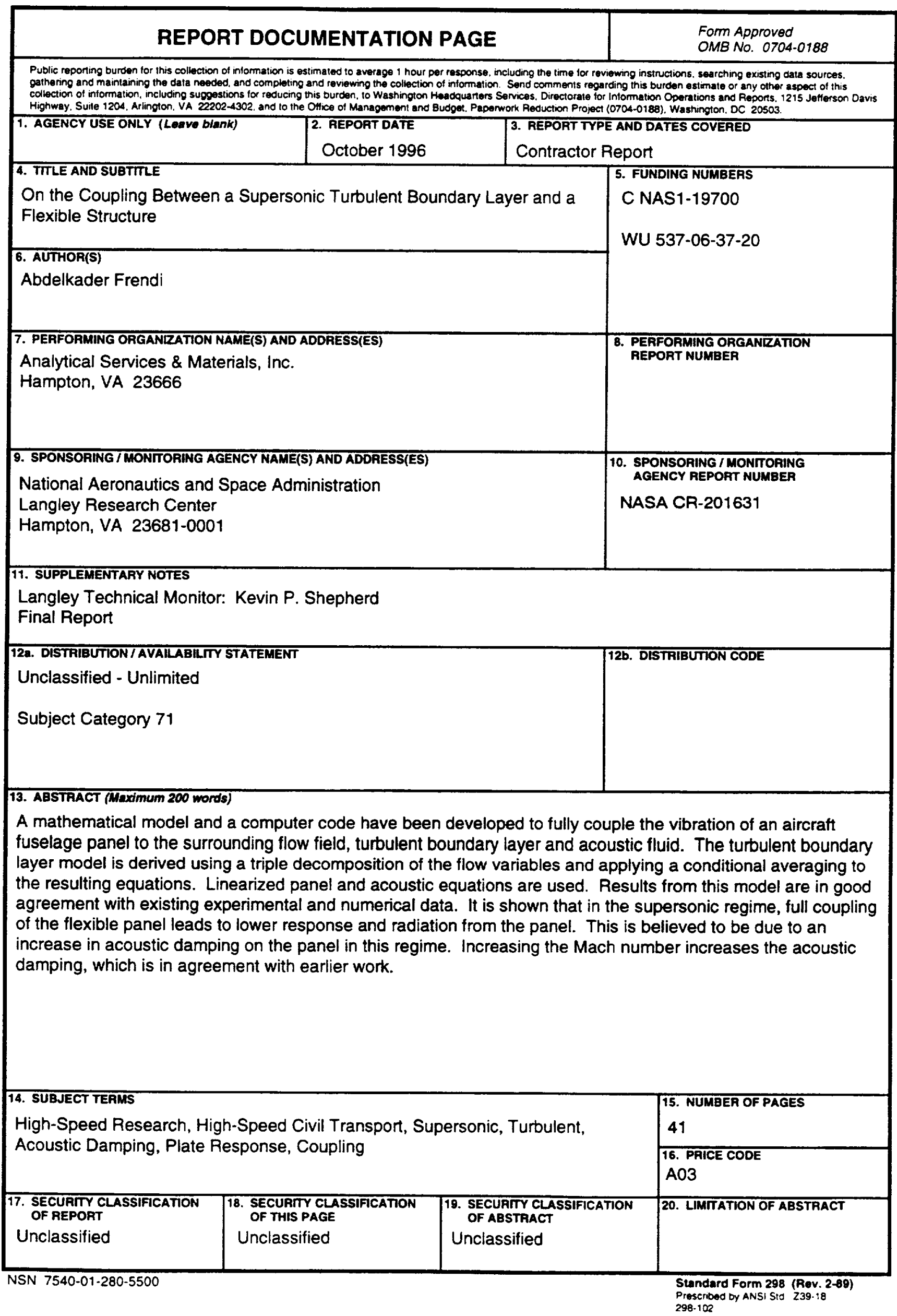

